\title{
Species diversity, distribution and habitat utilization of urban wildlife in a megacity of Bangladesh
}

\author{
Mohammad Firoj Jaman,,*, Abdur Razzaque Sarker', Mahabub Alam', Mokhlesur Rahmann',2, Fazle Rabbe ${ }^{1,3}$, \\ Abu Sayeed Rana',Ashikur Rahman Shome' \& Sakhawat Hossain ${ }^{4}$ \\ ${ }^{1}$ Department of Zoology, University of Dhaka, Dhaka 1000, Bangladesh \\ ${ }^{2}$ Department of Anthropology, Durham University, South Road, Durham DH1 3LE, UK \\ ${ }^{3}$ Padma Bridge Museum Project, Department of Zoology, University of Dhaka, Dhaka 1000, Bangladesh \\ ${ }^{4}$ Department of Zoology, Jagannath University, Dhaka 1100, Bangladesh \\ *Corresponding author, email: mfjaman4@gmail.com
}

\begin{abstract}
Urban environments harbour many species of wildlife. Such environments may provide a wide range of benefits, although these species could also face more threats in them than outside of the cities, without proper consideration. A comprehensive study of the wildlife in Dhaka, a megacity of Bangladesh, was conducted from September 2015 to November 2018 at eleven sites to estimate the extent of their presence. We collected data following transect line surveys for birds and mammals, and visual encounter surveys for herpetofauna. A total of 209 species belonging to 79 families of wildlife were recorded: 12 amphibians, 19 reptiles, 162 birds and 16 mammals. This study recorded a total of 13,805 individuals: 12,183 birds, 872 mammals, 605 amphibians, and 145 reptiles. In Shahbag site in total 3,039 individuals of wildlife were counted followed by Ramna $(2,576)$, Uttara $(2,108)$ and Mirpur $(1,872)$. Seventeen species were shared between habitats and an average $33 \%$ of the total species were shared between sites (range 13-52\%). Slightly more than half of the wildlife was recorded on trees, followed by grasslands and urban settlements. We identified some anthropogenic activities such as pollution, rapid construction work, random vehicle movements, using parks and gardens as a short passageway that may have a negative effect on urban wildlife distribution and survivability. Our baseline data on vertebrate wildlife diversity indicate that urban green patches in the study sites may contribute to maintain and conserve biodiversity in the megacities. We hope that the results of this baseline data on wildlife diversity will be valuable to urban decision makers for the development and implementation of more informed megacity master plans. Wildlife diversity in such areas can be significant and without proper planning, can be affected by unchecked human activities in urban settings.
\end{abstract}

KEY WORDS Habitat utilization; Species richness; Urban wildlife; Wildlife diversity.

Received 16.03.2021; accepted 29.06.2021; published online 14.09.2021

\section{INTRODUCTION}

Urbanization is seemingly the most prominent anthropogenic transformation that the ecological system experiences these days across the world (Rocha \& Fellowes, 2018). These human transformations have appeared to change the structure of landscape ecology, promoting homogenization of habitats, resulting in dramatic changes in biotic communities (McKinney, 2006). Usually urbanization has an effect on decreasing biodiversity, though there are some urban wildlife species living in such environments. Wildlife living in urban areas faces more stress than their counter-parts living outside of cities (Gibbs et al., 2019). However, there are also a range of benefits to living in urban 
habitats e.g., greater food availability (Jessop et al., 2012), less predation pressure (Rebolo-Ifran et al., 2017), and a refugium from many natural infectious diseases (Saenz et al., 2015). Urban habitats also create corridors for individuals that need to move through cities (FitzGibbon et al., 2007) and play an important ecological role in protecting local biodiversity by providing shelter (Crooks et al., 2004; Aronson et al., 2017).

Urban wildlife also provide important ecosystem services that benefit humans through the regulation of insect and rodent populations (Sekercioglu et al., 2004), seed dispersal and pollination (Sekercioglu et al., 2004; Mendes et al., 2008), removal of carcasses and nutrient cycling (Pain et al., 2003). They also promote economic benefits such as bird watching tourism (Sekercioglu, 2002) and an increase in property value (Bolitzer \& Netusil, 2000). Most importantly, urban wildlife is sharing the same habitats with urban citizens in the surrounding environments (Magle et al., 2012). Therefore, urban wildlife is now considered as a part of modern city life. For a functional urban ecosystem, it is important to maintain the balance between a healthy ecosystem and changes due to urbanization. In this light, because the knowledge gap about the importance of these groups of animals living among people may lead to conflicts in wildlife management (Kato et al., 2019), the study of urban animals, including their ecology, diversity, distribution and status is extremely important. Urban wildlife management is a new concept, compared to wildlife game management and damage control strategies (McCance et al., 2017). Therefore, baseline data on urban wildlife is essential for properly informed urban management planning in the future. Understanding the importance of animals in modern life, research on urban wildlife is increasing worldwide (Magle et al., 2012).

Studies on urban wildlife in developing Asian countries are few compared to the developed countries in North America and Europe (Magle et al., 2012). For instance, Dhaka, one of the oldest and biggest cities in Asia, does not have a comprehensive urban wildlife and conservation management plan for sustainable urban development. Dhaka is one of the fastest growing megacities in terms of urban transformation and subsequent environmental change (Islam \& Ahmed, 2011; Akash et al., 2018). There are several ongoing megaprojects (for example, metrorails, flyovers/multilayer roads) and recent expansion of the city at Uttara altered the natural habitats and changed the landscape structure that may eventually favorably affect urban wildlife. Furthermore, some recent findings from Dhaka city, such as the discovery of a new frog species, Fejervarya dhaka (Howlader et al., 2016), new bird colonies (Sahadat Hossain, pers.com. official of Bangladesh Bird Club) and some new locality records of reptile species (Sarker, 2013; Hasan et al., 2014), revealed this city to be an important new site for wildlife studies. On the other hand, Islam \& Ahmed (2011) recently showed that during the period from 1991-2008, 14.59\% of agricultural land, 16.92\% of grassy and bushy areas and some large and small trees in and around human settlements, $10.27 \%$ of waterbodies, and $1.28 \%$ wet/or lowlands were lost while $14.84 \%$ land covered with new residential and commercial buildings and infrastructures. All taken together, these facts suggest that Dhaka is a good model for studying urban wildlife. These studies in future may give the direction to better understanding the effects of urbanization on them.

Despite the importance and potential, research on wildlife diversity in Dhaka megacity is still underrepresented with only a handful numbers of articles published to date. Among these for instance are about the avifauna of Uttara (Sarker et al., 2009), avifauna of Dhaka University campus (Akash et al., 2013; Chowdhury et al., 2014; Banu et al., 2016), birds in the National Botanical Garden (NBG) (Islam et al., 2014), Keraniganj (Jaman et al., 2014), Ramna park (Rajia et al., 2015), some herpetological studies (Sarker, 2013; Hasan et al., 2014) and a dolphin survey in an adjacent river (Hossain \& Baki, 2015). The published literature indicates that the previous studies in Dhaka city were conducted on some scattered localities, mainly focusing on single taxa. In addition, there has been no single study recording everything from Amphibia to Mammalia in the whole of Dhaka city. Moreover, we identified some untouched areas not studied previously that indicate important gaps in our knowledge of the city's wildlife. Given that there appears to be a geographic bias and a limited focus on wildlife species studied within the megacity, the overall diversity, distribution, habitat use and conservation status of wildlife species in Dhaka city have not yet been fully documented. This study aimed to provide baseline data on overall urban wildlife diversity, patterns of species distribution, habitat use and current conservation status in the rapidly changing urbanized landscape of Dhaka 
city. This study also aimed to fill in the research gaps on wildlife diversity by considering all locations under this study.

\section{MATERIAL AND METHODS}

\section{Study sites and habitats surveyed}

The study was conducted from September 2015 to November 2018 at 11 sites in Dhaka city $\left(23.7^{\circ} \mathrm{N}\right.$, $90.4^{\circ} \mathrm{E}$ ), including parks, lakes, ponds, roadsides, homestead gardens, agricultural lands and public gardens (Table 1; Fig. 1). These study sites were selected based on the preliminary survey of the potential wildlife habitats and focused discussion with local stakeholders.

\section{Data collection}

During the survey, we collected information on the number of species, abundance, habitats and substrate utilization, as well as potential threats to wildlife. We spent 33 (11 locations $\times 3$ months)

\begin{tabular}{|c|c|}
\hline $\begin{array}{l}\text { Site name } \\
\left(\text { area } \mathbf{k m}^{2}\right)\end{array}$ & Habitat surveyed in the study areas \\
\hline $\begin{array}{l}\text { Agargaon } \\
(1.2)\end{array}$ & $\begin{array}{l}\text { Sher-e-Bangla Agricultural University (SAU) Campus: Gardens and agriculture experiment } \\
\text { fields, ponds, temporary waterbodies, roadside vegetation, building walls }\end{array}$ \\
\hline \multirow{2}{*}{$\begin{array}{l}\text { Demra } \\
(6.88)\end{array}$} & Mogdapara and Matuail: Human habitations, roadside trees, small waterbodies \\
\hline & Jatrabari: homestead areas, gardens and planted trees \\
\hline $\begin{array}{l}\text { Gulistan } \\
(0.26)\end{array}$ & Dhaka Mahanagar Nattomoncho Park trees, Osmani Park, Nagar Bhaban gardens \\
\hline $\begin{array}{l}\text { Gulshan } \\
(2.84)\end{array}$ & Gulshan park garden and lake waterbodies \\
\hline \multirow{2}{*}{$\begin{array}{l}\text { Mirpur } \\
(1.67)\end{array}$} & NBG: trees, grasslands and lake water \\
\hline & Dhaka Zoo: Planted trees, grasslands and lake water \\
\hline $\begin{array}{l}\text { Mohakhali } \\
(1.43)\end{array}$ & Shaheen College yards, Dhaka Cantonment gardens, Shahid Sarani gardens and trees \\
\hline \multirow[t]{2}{*}{$\begin{array}{l}\text { Mohammadpur } \\
(0.90)\end{array}$} & $\begin{array}{l}\text { Chandrima Udyan, Mohammadpur and Bosila: Residential area, planted trees, growing urban } \\
\text { areas }\end{array}$ \\
\hline & Rayer Bazar Graveyard: Around lakesides, roadside plants and building \\
\hline \multirow{2}{*}{$\begin{array}{l}\text { Old Dhaka } \\
(0.94)\end{array}$} & Buriganga river sides, trees, waterbodies \\
\hline & Balda Garden: Planted garden and trees \\
\hline \multirow{2}{*}{$\begin{array}{l}\text { Ramna } \\
(0.80)\end{array}$} & Ramna Park: Lakes, waterbodies, plants, roadside plants and grasslands \\
\hline & Baily Road homestead areas and roadside plants \\
\hline \multirow{7}{*}{$\begin{array}{l}\text { Shahbagh } \\
(1.87)\end{array}$} & University of Dhaka Campus: Garden plants, temporary waterbodies, pond, buildings \\
\hline & Suhrawardy Udyan: Plants, ponds, grasslands and temporary waterbodies \\
\hline & Buildings and Malchattar \\
\hline & North and South Fuller Road homestead plants \\
\hline & $\begin{array}{l}\text { Bangladesh University of Engineering and Technology (BUET) Campus: gardens, community } \\
\text { forest and university campus. }\end{array}$ \\
\hline & North \& south Fuller Road homestead areas \\
\hline & University of Dhaka Giasuddin Residential Area, trees \\
\hline $\begin{array}{l}\text { Uttara } \\
(14.21)\end{array}$ & $\begin{array}{l}\text { Uttara (Badda-Aftabnagar and Diabari): building walls, drains, roadside plantations, waterbodies, } \\
\text { grasslands }\end{array}$ \\
\hline
\end{tabular}

Table 1 . The habitats surveyed at different study sites during the study period. 


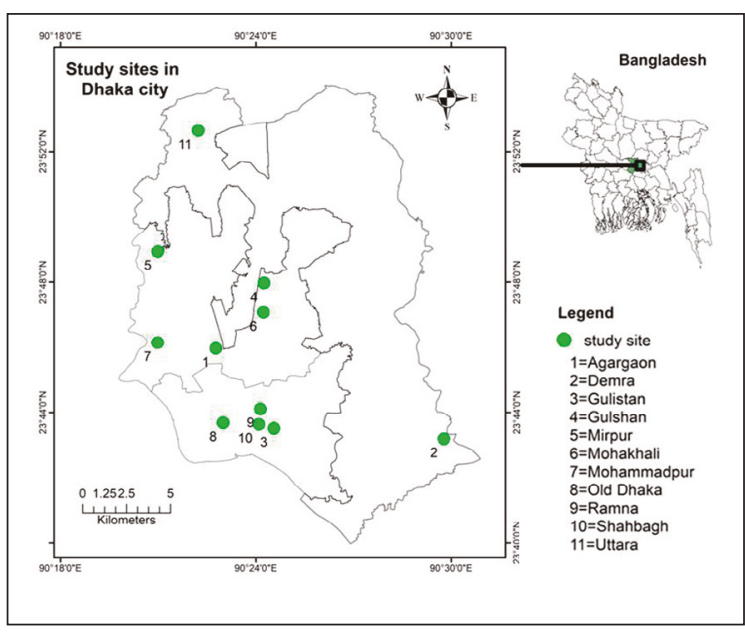

Figure 1. Study sites in Dhaka city.

months for data collection during the study period and visited each site at least three times, once each in summer, rainy season and winter. We surveyed 24 (3seasons $\times 8$ days) days per site totaling 264 (11 sites $\times 24$ days) days with equal effort for each group of wildlife. We surveyed approximately 160,000 square meters in each site; visited each transect line and remarked areas three times across seasons and used all methods (i.e. transect line survey for the bird and mammal, visual encounter survey - 'VES' of herpetofauna). We conducted diurnal and nocturnal surveys employing several methods to collect data on four different groups of wildlife. Species were primarily identified by direct observation and scrutinizing of photographs. We photographed the observed species by using DSLR cameras and Canon Power shot A2500. For species identification and nomenclature, we followed Halder (2010), Khan (2015) and Khan (2018) for birds; Khan (2004), Hasan et al. (2014), Khan (2018), Uetz et al. (2019) and Frost (2020) for herpetofauna and Khan $(2015,2018)$ for mammals.

Amphibians and Reptiles. We conducted VES randomly, but equally (in terms of area) in each site for herpetofauna survey in the day (from 10:00 am to 2:00 pm) and night (from 6:00 pm to 12:00 am). Nocturnal VES was most successful for amphibians and some nocturnal reptiles in the rainy season (Wheater et al., 2011). We walked along the trails across the habitats and actively looked for exposed or active animals. We used handheld flashlights and head torches during night observations
(Gent \& Gibson, 2003). We recorded vocalizations and analyzed them for the identification of cryptic frog species (Khan, 2004; Hasan et al., 2014; Khan, 2018; Uetz et al., 2019 and Frost, 2020). Lizards were counted while walking on the study site. In many cases, we searched for camouflaged herpetofauna in their preferred microhabitats (e.g., grassland, bushy areas, trees, buildings) and sometimes located them by their calls.

Birds. We counted avifauna populations using binoculars (Prism a 20×50) and recorded species using the line transect method from 5:30 am to 11:30 pm (van Heezik \& Seddon, 2012). Widths of each transect line varied from $10 \mathrm{~m}$ to $30 \mathrm{~m}$ in width and from $200 \mathrm{~m}$ to $1000 \mathrm{~m}$ in length, depending on the location of habitats we surveyed. Although transect number and total area varied in different study sites, we ensured covering similar volume of area in all selected sites. For example, to cover small fragmented areas like Gulisthan, we surveyed 32 transects totaling 160,000 square meters of varied sizes; 2 transects $(250 \times 10 \times 2$ square meter $), 20$ transects $(400 \times 10 \times 20$ square meter) and 10 transects $(500 \times 15 \times 10$ square meter). Whereas for Uttara, we surveyed 17 transects covering a similar areas we did for Gulisthan, but sizes of transects varied; 5 transects $(800 \times 20 \times 5$ square meter $), 2$ transects $(1000 \times 30 \times 2$ square meter) and 10 transects $(200 \times 10 \times 10$ square meter). We also surveyed at night for nocturnal birds from 6:00 pm to $12: 00 \mathrm{pm}$ and traced individuals by their calls. Sometimes we counted birds their calls and songs and in a few cases, we recorded calls and later identified them. We contacted dedicated birders of Dhaka city to know the breeding and roosting sites for migratory and colonial birds and later surveyed those areas.

Mammals. We collected data on mammals in the same transect lines used for birds at the same time. We opportunistically searched most of the natural habitats in transects to record mammals. We did nocturnal surveys for nocturnal carnivores and rodents from 6:00 pm to 12:00 pm (following Wheater et al., 2011); traced them by footprints, scat and fur traces. Aquatic and flying mammals were photographed and later identified. In some areas, we interviewed local people to get information on available mammalian species. We showed them photographs available in the field pictorial guides and confirmed species identification based on their descriptions. 


\section{Habitat survey}

We recorded urban wildlife from various microhabitats. These microhabitats are small recognizable areas within a habitat and were divided into Grassland (GL) and Tree (T), Roadside area (RA), Permanent waterbody (PW), Temporary waterbody (TW), Urban settlement (US), and Flying condition. Grassland includes grassy and bushy areas; Tree includes large and small trees in patchy areas, planted trees, gardens; Roadside area includes the land that is along a road; Permanent waterbody includes ponds, lakes, and river; Temporary waterbody includes pools, canals, small water bodies, and drains; and urban settlement includes buildings, construction sites, residential and commercial areas. Some wildlife, especially birds and flying mammals, were recorded in flight and were not observed resting in other habitat types. We recorded these habitat as "Flying". We classified substrates vertically as ground layers (animal counting from the ground), lower canopy ( $\leq 4 \mathrm{~m}$ height), middle canopy ( $>4 \mathrm{~m}$ $-<6 \mathrm{~m}$ height) and upper canopy (>6 m height).

\section{Data analysis}

Data collected for amphibians, reptiles, birds and mammals were analyzed in spreadsheets for diversity index, relative abundance, habitat, and substrate utilization in different sites. We compared the site-wise diversity index and evenness following the method of Shannon-Wiener index (Shannon \& Weaver, 1949). We also investigated the similarity of species composition in the sites studied. We calculated similarity indices for the eleven study sites using EstimateS software (Chao et al., 2005). We did not compare seasonal diversity as amphibians and reptiles were mostly observed in the rainy season, which would bias their seasonal diversity. We calculated the frequency of occurrence (n), which means how many times a particular species in each site, was observed. Relative abundance (RA) was calculated following Hasan et al., (2014) as very common (75-100\% of occurrence), common (51-74\% of occurrence), uncommon (26-50\% of occurrence) and rare ( $<26 \%$ of occurrence). [RA equation: number of observations of the species at different sites (n)/total number of observations (here, $11)^{*} 100$. If a species is seen in all study sites (11), then $\mathrm{RA}=11 / 11 * 100=100 \%$ (very common); if a species is seen in 5 out of 11 sites, then $\mathrm{RA}=5 / 11 * 100=45 \%$ (uncommon)]. Relative abundance was calculated to estimate the status of the observed species in Dhaka city. We also appended data of regional conservation status from the IUCN Red List Bangladesh (IUCN Bangladesh, 2015).

\section{RESULTS}

\section{Species diversity, composition and Shannon index}

A total of 209 species were recorded across the study sites (Table 3). Among them, 12 (5.74\%) species were amphibians, $19(9.09 \%)$ reptiles, 162 (77.5\%) birds and $16(7.66 \%)$ were mammals. These amphibians, reptiles, birds and mammals belonged to 5, 11, 52 and 11 families, respectively (Table 3). Among the total species observed, 65.6\%, (137) were in Uttara followed by $44.5 \%$ (93) in Mirpur, $26.3 \%$ (55) in Shahbagh, 23.9\% (50) in Old Dhaka and $20.6 \%$ (43) in Ramna (Fig. 2). According to taxa, the numbers of recorded species from different study sites are shown in Fig. 2.

A total of 13,805 individuals were counted from the study sites (Table 3). Among them, birds were the highest in number $(88 \%, 12,183)$ followed by mammals $(6.3 \%, 872)$, amphibians $(4.4 \%, 605)$, and reptilians $(1.05 \%, 145$, Table 3$)$. Regarding the sitewise total count of individuals, the highest number of individuals was counted at Shahbag $(22 \%, 3,039)$ followed by Ramna (18.7\%, 2,576), Uttara (15.3\%, $2,108)$ and Mirpur $(13.6 \%, 1,872)$ (Table 3).

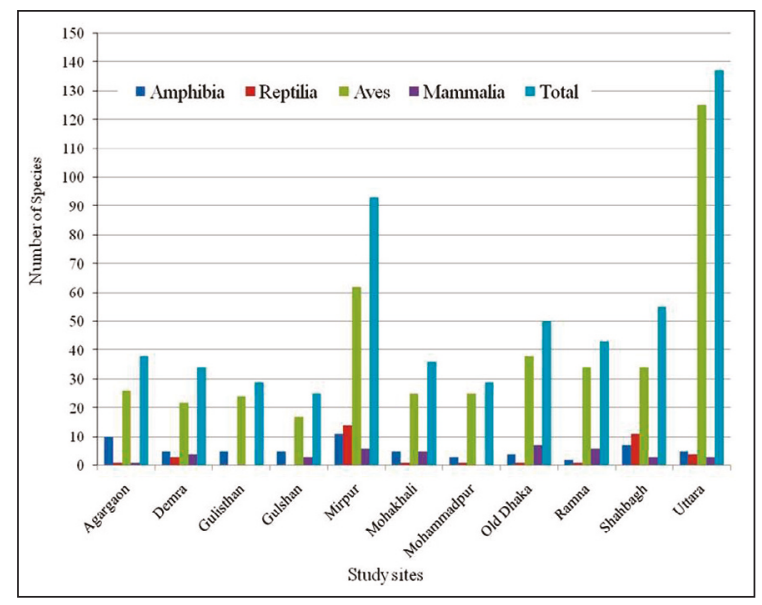

Figure 2. Species richness of wildlife recorded at different study sites of urban Dhaka megacity. 


\begin{tabular}{|l|c|c|c|c|c|c|c|c|c|c|c|}
\hline Parameter & Site 1 & Site 2 & Site 3 & Site 4 & Site 5 & Site 6 & Site 7 & Site 8 & Site 9 & Site 10 & Site 11 \\
\hline $\begin{array}{l}\text { Shannon-Wiener } \\
\text { Index }\left(\mathrm{H}^{\prime}\right)\end{array}$ & 3.350 & 2.871 & 2.003 & 2.962 & 3.647 & 2.555 & 2.734 & 3.077 & 2.720 & 2.829 & 4.198 \\
\hline Evenness $(\mathrm{E})$ & 0.921 & 0.814 & 0.595 & 0.920 & 0.805 & 0.713 & 0.812 & 0.786 & 0.723 & 0.706 & 0.853 \\
\hline
\end{tabular}

Table 2. Shannon-Wiener indices listed by the study site. (Site 1- Agargaon; Site 2- Demra; Site 3- Gulistan; Site 4- Gulshan; Site 5- Mirpur; Site 6- Mohakhali; Site 7-Mohammadpur; Site 8- Old Dhaka; Site 9-Ramna; Site 10-Shahbag, and Site 11Uttara).

The highest value of Shannon-Wiener index was calculated $\left(\mathrm{H}^{\prime}=4.198\right)$ for site no.11 (Uttara), followed by 3.64 for site 5 (Mirpur), 3.35 for site 1 (Agargaon), 3.07 for site 8 (Old Dhaka) (Table 2). Species were more evenly distributed in the Agargaon site $(\mathrm{E}=0.921)$, probably due to the abundance of homestead trees that are lacking in Gulistan $(\mathrm{E}=$ 0.595) (Table 2).

\section{Relative abundance}

Among the total species of wildlife, 20 (9.6\%) were very common, 16 (7.7\%) common, $20(9.6 \%)$ uncommon and $153(73.2 \%)$ species were rare (Table $3)$. Among amphibians, 2 (16.7\%) species were very common, 4 (33.3\%) common, 3 (25\%) uncommon, and $3(25 \%)$ species were rare (Table 3$)$. Among reptiles, $18(94.7 \%)$ species were rare and the remaining one $(5.3 \%)$ species was common. Among 162 species of birds, $120(74.1 \%)$ were rare, 16 (9.9\%) were uncommon, 15 (9.3\%) were very common and $11(6.8 \%)$ were common. Of the recorded mammals, 12 (75\%) species were rare, 3 (18.8\%) very common and $1(6.3 \%)$ species was uncommon in Dhaka city (Table 3).

\section{Species similarity index}

Some species shared different habitats with other species. The similarity index indicates the sharing of habitats by the number of species recorded. The calculated index values range from 0.13 to 0.52 (average $=0.33$ ) for pair-wise study sites, which means that on average $33 \%$ of the recorded species were common in all eleven sites (Table 3).

\section{Habitat and substrate utilization}

Species were the most abundant in trees than in other microhabitats; those being mostly birds. When comparing overall species in microhabitat types, 117

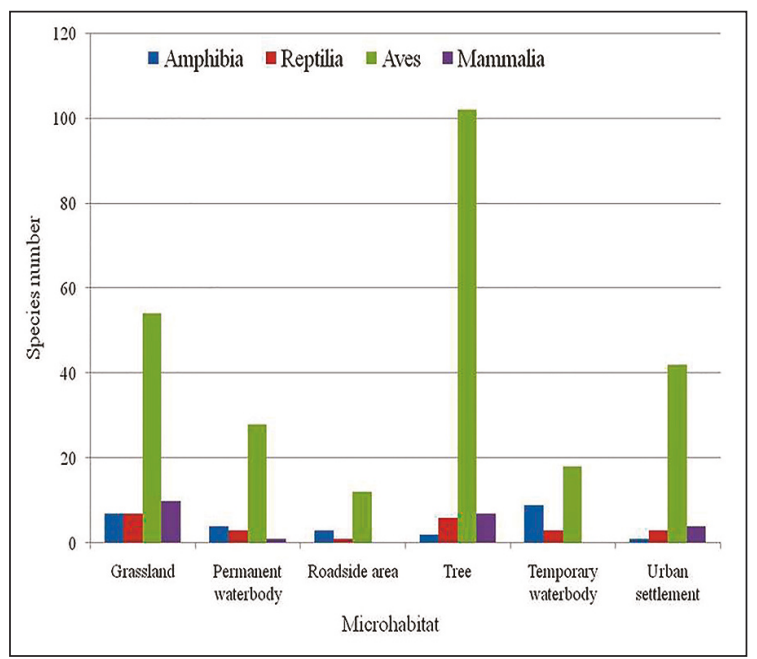

Figure 3. Microhabitat usage of wildlife in urban Dhaka city.

species were found in trees followed by 78 in grassland, 50 in urban settlements, 36 on permanent waterbodies, 30 on temporary waterbodies (Fig. 3).

Amphibians were mostly recorded from permanent and temporary waterbodies and near its grasslands. Seven species of reptiles used grassland, which was their most frequently used microhabitat. Among birds, the highest numbers of species (102) were recorded in trees and the lowest (3) were observed while flying. For mammals, the highest (10) species were found in grasslands.

All wildlife species were found using different vertical layers of habitat and they frequently move in different canopy levels for foraging and displaying other activities. Among them, 118 species used ground layers, which was the highest followed by 84 species in middle canopy, 71 in upper canopy, 62 in lower canopy and 56 in man height layer (Fig. 4). We found 17 species that utilized all substrate types (Fig. 4). The unique species number observed for different substrates is 72 for ground layer, 8 for lower canopy, 21 for middle canopy, 19 for upper canopy, and 7 for man height layer (Fig. 4). 


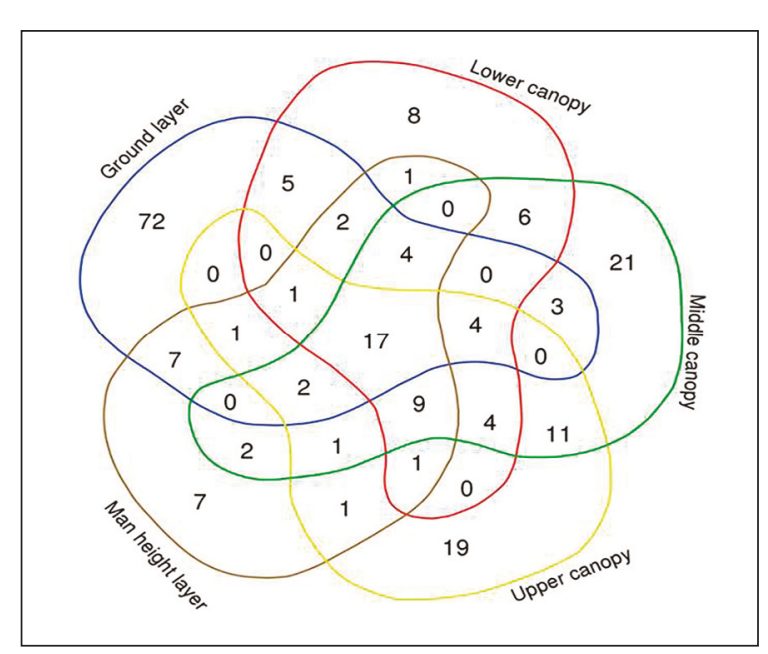

Figure 4. Substrate utilization of wild animals in Dhaka city (number denoted the species those utilized and shared single and or multiple substrates).

\section{Threatened status}

According to IUCN Bangladesh (2015), among the 209 species of wildlife listed here, eight were Near Threatened (three reptiles, two birds and three mammals), two species Vulnerable (mammals), two species Data Deficient (bird), and 197 species were Least Concern (Table 3).

\section{DISCUSSION}

\section{Species composition, abundance and distribution}

Urban habitats sometimes provide benefits for wildlife such as stable food availability, absence of natural predators and even green corridors for animals to move or migrate through, playing a vital ecological role in protecting local biodiversity. Our study has provided substantial baseline data on diverse taxa of wildlife with a significant number of different species, distribution and habitat utilization in the urbanized Dhaka megacity. The most diverse amphibian species were found in the family Dicroglossidae, having $8(67 \%)$ species of frogs. The most frequently observed species was the Asian Common Toad $(21.2 \%, 128$ indiv.) and the least common was the Green Frog (0.33\%, 2 indiv.), sighted only in NBG. Some amphibians were recorded only from one site. Of the recorded amphibians, the Marbled Toad, Jerdon's Bull Frog, and Balloon Frog were not observed in this study but were recorded earlier by Reza \& Perry (2015). They reported herpetofaunal species occurrence using both direct observation and literature searching while we only used VES for this study. For this reason, we found fewer species of herpetofauna

\begin{tabular}{|c|c|c|c|c|c|c|c|}
\hline $\begin{array}{l}\text { Order and } \\
\text { Family name }\end{array}$ & Common Name & Scientific Name & $\mathbf{n}$ & $\begin{array}{c}\text { No. of } \\
\text { Individual }\end{array}$ & RA & $\begin{array}{l}\text { IUCN } \\
\text { Status }\end{array}$ & Microhabitat \\
\hline \multicolumn{8}{|c|}{ Amphibia } \\
\hline \multirow[t]{9}{*}{$\begin{array}{l}\text { Anura } \\
\text { Bufonidae }\end{array}$} & $\begin{array}{l}\text { Asian Common } \\
\text { Toad }\end{array}$ & $\begin{array}{l}\text { Duttaphrynus } \\
\text { melanostictus }\end{array}$ & 11 & 128 & $\mathrm{VC}$ & LC & $\begin{array}{c}\text { GL, PW, RS, } \\
\text { TW, US }\end{array}$ \\
\hline & Indian Bull Frog & $\begin{array}{l}\text { Hoplobatrachus } \\
\text { tigerinus }\end{array}$ & 10 & 60 & $\mathrm{C}$ & $\mathrm{LC}$ & GL, TW \\
\hline & Green Frog & $\begin{array}{l}\text { Euphlyctis } \\
\text { hexadactylus }\end{array}$ & 1 & 2 & $\mathrm{R}$ & $\mathrm{LC}$ & PW \\
\hline & Skipper Frog & E. cyanophlyctis & 10 & 68 & $\mathrm{C}$ & $\mathrm{LC}$ & PW, TW \\
\hline & $\begin{array}{l}\text { Kalasgram } \\
\text { Skipper Frog }\end{array}$ & E. kalasgramensis & 1 & 7 & $\mathrm{R}$ & $\mathrm{LC}$ & $\mathrm{PW}$ \\
\hline & $\begin{array}{l}\text { Pierre's Cricket } \\
\text { Frog }\end{array}$ & Fejervarya pierrei & 2 & 29 & $\mathrm{UC}$ & $\mathrm{LC}$ & GL, TW \\
\hline & $\begin{array}{l}\text { Asmat's Cricket } \\
\text { Frog }\end{array}$ & F. asmati & 6 & 90 & $\mathrm{VC}$ & LC & GL, TW \\
\hline & $\begin{array}{l}\text { Bombay Wart } \\
\text { Frog }\end{array}$ & F. syhadrensis & 6 & 71 & $\mathrm{C}$ & $\mathrm{LC}$ & GL, RA, TW \\
\hline & Terai Cricket Frog & F. teraiensis & 7 & 74 & $\mathrm{C}$ & $\mathrm{LC}$ & GL, TW \\
\hline Rhacophoridae & $\begin{array}{l}\text { Common Tree } \\
\text { Frog }\end{array}$ & $\begin{array}{l}\text { Polypedates } \\
\text { leucomystax }\end{array}$ & 4 & 27 & UC & LC & $\mathrm{T}$ \\
\hline
\end{tabular}




\begin{tabular}{|c|c|c|c|c|c|c|c|}
\hline $\begin{array}{l}\text { Order and } \\
\text { Family name }\end{array}$ & Common Name & Scientific Name & $\mathbf{n}$ & $\begin{array}{c}\text { No. of } \\
\text { Individual }\end{array}$ & $\mathbf{R A}$ & $\begin{array}{l}\text { IUCN } \\
\text { Status } \\
\end{array}$ & Microhabitat \\
\hline Microhylidae & $\begin{array}{l}\text { Narrow-mouthed } \\
\text { Frog }\end{array}$ & Microhyla sp. & 3 & 44 & $\mathrm{UC}$ & $\mathrm{LC}$ & GL, RS, TW \\
\hline Ranidae & $\begin{array}{l}\text { Yellow-striped } \\
\text { Frog }\end{array}$ & Hylarana tytleri & 1 & 5 & $\mathrm{R}$ & $\mathrm{LC}$ & PW, T \\
\hline \multicolumn{8}{|c|}{ Reptilia } \\
\hline $\begin{array}{l}\text { Squamata } \\
\text { Varanidae }\end{array}$ & Bengal Monitor & $\begin{array}{l}\text { Varanus } \\
\text { bengalensis }\end{array}$ & 2 & 12 & $\mathrm{R}$ & NT & GL, RS \\
\hline \multirow[t]{2}{*}{ Elapidae } & Monocled Cobra & Naja kaouthia & 3 & 3 & $\mathrm{R}$ & NT & GL \\
\hline & Binocled Cobra & N. naja & 3 & 6 & $\mathrm{R}$ & NT & GL \\
\hline \multirow[t]{2}{*}{ Natricidae } & $\begin{array}{l}\text { Checkered } \\
\text { Keelback }\end{array}$ & $\begin{array}{l}\text { Xenochrophis } \\
\text { piscator }\end{array}$ & 3 & 4 & $\mathrm{R}$ & $\mathrm{LC}$ & PW, TW \\
\hline & Striped Keelback & $\begin{array}{l}\text { Amphiesma } \\
\text { stolatum }\end{array}$ & 1 & 1 & $\mathrm{R}$ & $\mathrm{LC}$ & PW \\
\hline Typhlopidae & $\begin{array}{l}\text { Brahminy Blind } \\
\text { Snake }\end{array}$ & $\begin{array}{l}\text { Ramphotyphlops } \\
\text { braminus }\end{array}$ & 2 & 4 & $\mathrm{R}$ & $\mathrm{LC}$ & GL \\
\hline \multirow[t]{4}{*}{ Gekkonidae } & $\begin{array}{l}\text { Common House } \\
\text { Gecko }\end{array}$ & $\begin{array}{l}\text { Hemidactylus } \\
\text { frenatus }\end{array}$ & 3 & 25 & $\mathrm{R}$ & $\mathrm{LC}$ & $\mathrm{T}, \mathrm{US}$ \\
\hline & $\begin{array}{l}\text { Yellow-green } \\
\text { House Lizard }\end{array}$ & H. flaviviridis & 4 & 54 & $\mathrm{C}$ & $\mathrm{LC}$ & $\mathrm{T}, \mathrm{US}$ \\
\hline & $\begin{array}{l}\text { Brook's House } \\
\text { Gecko }\end{array}$ & H. brookii & 2 & 12 & $\mathrm{R}$ & $\mathrm{LC}$ & US \\
\hline & Tokay Gecko & Gekko gecko & 1 & 1 & $\mathrm{R}$ & $\mathrm{LC}$ & $\mathrm{T}$ \\
\hline \multirow[t]{2}{*}{ Scincidae } & Common Skink & Eutropis carinata & 1 & 1 & $\mathrm{R}$ & $\mathrm{LC}$ & GL \\
\hline & $\begin{array}{l}\text { White-spotted } \\
\text { Supple Skink }\end{array}$ & $\begin{array}{l}\text { Lygosoma } \\
\text { albopunctata }\end{array}$ & 1 & 1 & $\mathrm{R}$ & $\mathrm{LC}$ & GL \\
\hline \multirow[t]{3}{*}{ Colubridae } & $\begin{array}{l}\text { Common Wolf } \\
\text { Snake }\end{array}$ & Lycodon aulicus & 1 & 1 & $\mathrm{R}$ & $\mathrm{LC}$ & $\mathrm{T}$ \\
\hline & $\begin{array}{l}\text { Common } \\
\text { Bronzeback Tree } \\
\text { Snake } \\
\end{array}$ & $\begin{array}{l}\text { Dendrelaphis } \\
\text { tristis }\end{array}$ & 1 & 1 & $\mathrm{R}$ & $\mathrm{LC}$ & $\mathrm{T}$ \\
\hline & Indian Rat Snake & Ptyas mисоsa & 3 & 9 & $\mathrm{R}$ & $\mathrm{LC}$ & GL \\
\hline Agamidae & $\begin{array}{l}\text { Common Garden } \\
\text { Lizard }\end{array}$ & Calotes versicolor & 1 & 4 & $\mathrm{R}$ & $\mathrm{LC}$ & $\mathrm{T}$ \\
\hline Homalopsidae & $\begin{array}{l}\text { Smooth water } \\
\text { Snake }\end{array}$ & Enhydris enhydris & 2 & 2 & $\mathrm{R}$ & $\mathrm{LC}$ & PW \\
\hline $\begin{array}{l}\text { Testudines } \\
\text { Geomydidae }\end{array}$ & Roofed Turtle & Pangshura tecta & 1 & 4 & $\mathrm{R}$ & $\mathrm{LC}$ & TW \\
\hline \multicolumn{8}{|c|}{ Aves } \\
\hline \multirow[t]{6}{*}{$\begin{array}{l}\text { Accipitriformes } \\
\text { Accipitridae }\end{array}$} & $\begin{array}{l}\text { Long-legged } \\
\text { Buzzard }\end{array}$ & Buteo rufinus & 1 & 5 & $\mathrm{R}$ & $\mathrm{LC}$ & $\mathrm{T}$ \\
\hline & Shikra & Accipiter badius & 2 & 3 & $\mathrm{R}$ & $\mathrm{LC}$ & $\mathrm{T}$ \\
\hline & Black Kite & Milvus migrans & 10 & 780 & $\mathrm{VC}$ & $\mathrm{LC}$ & Flying, GL, T \\
\hline & Black-winged Kite & Elanus caeruleus & 3 & 5 & $\mathrm{UC}$ & $\mathrm{LC}$ & $\mathrm{T}, \mathrm{US}$ \\
\hline & Brahminy Kite & Haliastur indus & 4 & 17 & $\mathrm{UC}$ & $\mathrm{LC}$ & $\mathrm{T}, \mathrm{US}$ \\
\hline & $\begin{array}{l}\text { Crested Serpent } \\
\text { Eagle }\end{array}$ & Spilornis cheela & 1 & 1 & $\mathrm{R}$ & $\mathrm{LC}$ & US \\
\hline Pandionidae & Osprey & Pandion haliaetus & 1 & 3 & $\mathrm{R}$ & $\mathrm{LC}$ & US \\
\hline $\begin{array}{l}\text { Anseriformes } \\
\text { Anatidae }\end{array}$ & $\begin{array}{l}\text { Lesser Whistling } \\
\text { Duck }\end{array}$ & $\begin{array}{l}\text { Dendrocygna } \\
\text { javanica }\end{array}$ & 1 & 2 & $\mathrm{R}$ & $\mathrm{LC}$ & PW \\
\hline $\begin{array}{l}\text { Bucerotiformes } \\
\text { Upupidae }\end{array}$ & Common Hoopoe & Upupa epops & 1 & 2 & $\mathrm{R}$ & LC & GL \\
\hline
\end{tabular}




\begin{tabular}{|c|c|c|c|c|c|c|c|}
\hline $\begin{array}{l}\text { Order and } \\
\text { Family name }\end{array}$ & Common Name & Scientific Name & $\mathbf{n}$ & $\begin{array}{c}\text { No. of } \\
\text { Individual }\end{array}$ & $\mathbf{R A}$ & $\begin{array}{l}\text { IUCN } \\
\text { Status }\end{array}$ & Microhabitat \\
\hline \multirow{2}{*}{$\begin{array}{l}\text { Coraciformes } \\
\text { Coraciidae }\end{array}$} & Indochinese Roller & Coracias affinis & 1 & 1 & $\mathrm{R}$ & $\mathrm{LC}$ & US \\
\hline & Indian Roller & C. benghalensis & 1 & 9 & $\mathrm{R}$ & $\mathrm{LC}$ & $\mathrm{T}, \mathrm{US}$ \\
\hline Meropidae & Green Bee-eater & Merops orientalis & 1 & 2 & $\mathrm{R}$ & $\mathrm{LC}$ & $\mathrm{T}$ \\
\hline \multirow[t]{4}{*}{ Alcedinidae } & $\begin{array}{l}\text { Common } \\
\text { Kingfisher }\end{array}$ & Alcedo atthis & 8 & 39 & $\mathrm{C}$ & $\mathrm{LC}$ & GL, PW, T \\
\hline & Pied Kingfisher & Ceryle rudis & 1 & 11 & $\mathrm{R}$ & $\mathrm{LC}$ & $\mathrm{T}, \mathrm{US}$ \\
\hline & $\begin{array}{l}\text { Stork-billed } \\
\text { Kingfisher }\end{array}$ & $\begin{array}{l}\text { Pelargopsis } \\
\text { capensis }\end{array}$ & 2 & 16 & $\mathrm{R}$ & $\mathrm{LC}$ & PW, T \\
\hline & $\begin{array}{l}\text { White-breasted } \\
\text { Kingfisher }\end{array}$ & $\begin{array}{l}\text { Halcyon } \\
\text { smyrnensis }\end{array}$ & 10 & 102 & $\mathrm{VC}$ & $\mathrm{LC}$ & PW, T, US \\
\hline \multirow[t]{2}{*}{$\begin{array}{l}\text { Caprimulgiformes } \\
\text { Apodidae }\end{array}$} & Asian Palm Swift & $\begin{array}{l}\text { Cypsiurus } \\
\text { balasiensis }\end{array}$ & 6 & 329 & $\mathrm{C}$ & $\mathrm{LC}$ & Flying, GL, T \\
\hline & House Swift & Apus nipalensis & 8 & 586 & $\mathrm{C}$ & $\mathrm{LC}$ & Flying, T, US \\
\hline Caprimulgidae & $\begin{array}{l}\text { Large-tailed } \\
\text { Nightjar }\end{array}$ & $\begin{array}{l}\text { Caprimulgus } \\
\text { macrurus }\end{array}$ & 1 & 3 & $\mathrm{R}$ & $\mathrm{LC}$ & $\mathrm{T}$ \\
\hline \multirow[t]{4}{*}{$\begin{array}{l}\text { Charadriformes } \\
\text { Charadridae }\end{array}$} & Kentish Plover & $\begin{array}{l}\text { Charadrius } \\
\text { alexandrinus }\end{array}$ & 1 & 2 & $\mathrm{R}$ & $\mathrm{LC}$ & TW \\
\hline & $\begin{array}{l}\text { Little Ringed } \\
\text { Plover }\end{array}$ & C. dubius & 1 & 156 & $\mathrm{R}$ & $\mathrm{LC}$ & PW, TW \\
\hline & $\begin{array}{l}\text { Red-wattled } \\
\text { Lapwing }\end{array}$ & Vanellus indicus & 1 & 5 & $\mathrm{R}$ & $\mathrm{LC}$ & GL, TW \\
\hline & River Lapwing & V. duvaucelii & 1 & 5 & $\mathrm{R}$ & NT & PW \\
\hline Jacanidae & $\begin{array}{l}\text { Bronze-winged } \\
\text { Jacana }\end{array}$ & $\begin{array}{l}\text { Metopidius } \\
\text { indicus }\end{array}$ & 2 & 3 & $\mathrm{R}$ & $\mathrm{LC}$ & PW \\
\hline Rostratulidae & $\begin{array}{l}\text { Greater Painted } \\
\text { Snipe }\end{array}$ & $\begin{array}{l}\text { Rostratula } \\
\text { benghalensis }\end{array}$ & 1 & 2 & $\mathrm{R}$ & $\mathrm{LC}$ & GL \\
\hline \multirow[t]{6}{*}{ Scolopacidae } & Wood Sandpiper & Tring a glareola & 1 & 2 & $\mathrm{R}$ & $\mathrm{LC}$ & TW \\
\hline & $\begin{array}{l}\text { Common } \\
\text { Sandpiper }\end{array}$ & Actitis hypoleucos & 1 & 103 & $\mathrm{R}$ & $\mathrm{LC}$ & PW, TW \\
\hline & Common Snipe & $\begin{array}{l}\text { Gallinago } \\
\text { gallinago }\end{array}$ & 1 & 2 & $\mathrm{R}$ & $\mathrm{LC}$ & PW \\
\hline & Pin-tailed Snipe & G. stenura & 1 & 2 & $\mathrm{R}$ & $\mathrm{LC}$ & PW \\
\hline & Little Stint & Calidris minuta & 1 & 4 & $\mathrm{R}$ & $\mathrm{LC}$ & TW \\
\hline & Temminck's Stint & C. temminckii & 1 & 8 & $\mathrm{R}$ & $\mathrm{LC}$ & TW \\
\hline \multirow[t]{4}{*}{$\begin{array}{l}\text { Psittaciformes } \\
\text { Psittacidae }\end{array}$} & $\begin{array}{l}\text { Alexandrine } \\
\text { parakeet }\end{array}$ & $\begin{array}{l}\text { Psittacula } \\
\text { eupatria }\end{array}$ & 4 & 13 & $\mathrm{UC}$ & $\mathrm{LC}$ & $\mathrm{T}$ \\
\hline & $\begin{array}{l}\text { Plum-headed } \\
\text { Parakeet }\end{array}$ & P. cyanocephala & 1 & 3 & $\mathrm{R}$ & LC & US \\
\hline & $\begin{array}{l}\text { Red-breasted } \\
\text { Parakeet }\end{array}$ & P. alexandri & 1 & 2 & $\mathrm{R}$ & $\mathrm{LC}$ & $\mathrm{T}$ \\
\hline & $\begin{array}{l}\text { Rose-ringed } \\
\text { Parakeet }\end{array}$ & P. krameri & 10 & 224 & $\mathrm{VC}$ & $\mathrm{LC}$ & $\mathrm{T}, \mathrm{US}$ \\
\hline \multirow{3}{*}{$\begin{array}{l}\text { Falconiformes } \\
\text { Falconidae }\end{array}$} & Amur Falcon & Falco amurensis & 1 & 1 & $\mathrm{R}$ & $\mathrm{LC}$ & US \\
\hline & $\begin{array}{l}\text { Red-headed } \\
\text { Falcon }\end{array}$ & F. chicquera & 2 & 3 & $\mathrm{R}$ & $\mathrm{LC}$ & $\mathrm{T}$ \\
\hline & Common Kestrel & F. tinnunculus & 1 & 8 & $\mathrm{R}$ & $\mathrm{LC}$ & $\mathrm{T}, \mathrm{US}$ \\
\hline \multirow[t]{3}{*}{$\begin{array}{l}\text { Passeriformes } \\
\text { Dicruridae }\end{array}$} & Ashy Drongo & $\begin{array}{l}\text { Dicrurus } \\
\text { leucophaeus }\end{array}$ & 1 & 4 & $\mathrm{R}$ & $\mathrm{LC}$ & US \\
\hline & Black Drongo & D. macrocercus & 11 & 208 & $\mathrm{VC}$ & $\mathrm{LC}$ & GL, RS, T,US \\
\hline & Bronzed Drongo & D. aeneus & 1 & 2 & $\mathrm{R}$ & $\mathrm{LC}$ & US \\
\hline
\end{tabular}




\begin{tabular}{|c|c|c|c|c|c|c|c|}
\hline $\begin{array}{l}\text { Order and } \\
\text { Family name } \\
\end{array}$ & Common Name & Scientific Name & $\mathbf{n}$ & $\begin{array}{c}\text { No. of } \\
\text { Individual }\end{array}$ & RA & $\begin{array}{l}\text { IUCN } \\
\text { Status } \\
\end{array}$ & Microhabitat \\
\hline & $\begin{array}{l}\text { Lesser Racket- } \\
\text { tailed } \\
\text { Drongo }\end{array}$ & D. remifer & 1 & 1 & $\mathrm{R}$ & $\mathrm{LC}$ & US \\
\hline Artamidae & $\begin{array}{l}\text { Ashy } \\
\text { Woodswallow }\end{array}$ & Artamus fuscus & 1 & 3 & $\mathrm{R}$ & $\mathrm{LC}$ & US \\
\hline Aegithinidae & Common Iora & Aegithina tiphia & 2 & 11 & $\mathrm{R}$ & $\mathrm{LC}$ & $\mathrm{T}$ \\
\hline \multirow[t]{2}{*}{ Alaudidae } & $\begin{array}{l}\text { Greater Short-toed } \\
\text { Lark }\end{array}$ & $\begin{array}{l}\text { Calandrella } \\
\text { brachydactyla }\end{array}$ & 1 & 15 & $\mathrm{R}$ & $\mathrm{DD}$ & GL \\
\hline & Bengal Bush Lark & Mirafra assamica & 1 & 9 & $\mathrm{R}$ & $\mathrm{LC}$ & GL \\
\hline \multirow[t]{4}{*}{ Campephagidae } & $\begin{array}{l}\text { Black-headed } \\
\text { Cuckooshrike }\end{array}$ & $\begin{array}{l}\text { Coracina } \\
\text { melanoptera }\end{array}$ & 1 & 2 & $\mathrm{R}$ & $\mathrm{LC}$ & $\mathrm{T}$ \\
\hline & $\begin{array}{l}\text { Black-winged } \\
\text { Cuckooshrike }\end{array}$ & C. melaschistos & 1 & 1 & $\mathrm{R}$ & $\mathrm{LC}$ & $\mathrm{T}$ \\
\hline & Small Minivet & $\begin{array}{l}\text { Pericrocotus } \\
\text { cinnamomeus }\end{array}$ & 1 & 1 & $\mathrm{R}$ & $\mathrm{LC}$ & US \\
\hline & Large Woodshrike & $\begin{array}{l}\text { Tephrodornis } \\
\text { gularis }\end{array}$ & 1 & 5 & $\mathrm{R}$ & $\mathrm{LC}$ & GL \\
\hline \multirow[t]{3}{*}{ Cisticolidae } & Plain Prinia & Prinia inornata & 4 & 45 & $\mathrm{UC}$ & $\mathrm{LC}$ & GL, T \\
\hline & $\begin{array}{l}\text { Grey-breasted } \\
\text { Prinia }\end{array}$ & P. hodgsonii & 1 & 4 & $\mathrm{R}$ & $\mathrm{LC}$ & PW \\
\hline & Zitting Cisticola & Cisticola juncidis & 5 & 140 & $\mathrm{UC}$ & $\mathrm{LC}$ & GL, RS, T, US \\
\hline \multirow[t]{3}{*}{ Corvidae } & Rufous Treepie & $\begin{array}{l}\text { Dendrocitta } \\
\text { vagabunda }\end{array}$ & 5 & 38 & $\mathrm{UC}$ & $\mathrm{LC}$ & $\mathrm{T}$ \\
\hline & Large-billed Crow & $\begin{array}{l}\text { Corvus } \\
\text { levaillantii }\end{array}$ & 8 & 275 & $\mathrm{C}$ & $\mathrm{LC}$ & GL, RS, T, US \\
\hline & House Crow & C. splendens & 11 & 2050 & $\mathrm{VC}$ & $\mathrm{LC}$ & GL, RS, T, US \\
\hline Dicaeidae & $\begin{array}{l}\text { Pale-billed } \\
\text { Flowerpecker }\end{array}$ & $\begin{array}{l}\text { Dicaeum } \\
\text { erythrorhynchos }\end{array}$ & 1 & 3 & $\mathrm{R}$ & $\mathrm{LC}$ & $\mathrm{T}$ \\
\hline \multirow[t]{6}{*}{ Estrildidae } & Chestnut Munia & $\begin{array}{l}\text { Lonchura } \\
\text { atricapilla }\end{array}$ & 3 & 60 & $\mathrm{UC}$ & $\mathrm{LC}$ & GL, T \\
\hline & $\begin{array}{l}\text { Scaly-breasted } \\
\text { Munia }\end{array}$ & L. punctulata & 1 & 12 & $\mathrm{R}$ & $\mathrm{LC}$ & GL, T \\
\hline & Tricolored Munia & L. malacca & 1 & 5 & $\mathrm{R}$ & $\mathrm{LC}$ & GL \\
\hline & $\begin{array}{l}\text { White-rumped } \\
\text { Munia }\end{array}$ & L. striata & 1 & 10 & $\mathrm{R}$ & $\mathrm{LC}$ & $\mathrm{T}$ \\
\hline & $\begin{array}{l}\text { White-throated } \\
\text { Munia }\end{array}$ & L. malabarica & 2 & 23 & $\mathrm{R}$ & $\mathrm{LC}$ & GL \\
\hline & Red Avadavat & $\begin{array}{l}\text { Amandava } \\
\text { amandava }\end{array}$ & 1 & 15 & $\mathrm{R}$ & $\mathrm{LC}$ & GL \\
\hline \multirow[t]{2}{*}{ Hirundinidae } & Barn Swallow & Hirundo rustica & 2 & 21 & $\mathrm{R}$ & $\mathrm{LC}$ & US \\
\hline & $\begin{array}{l}\text { Red-rumped } \\
\text { Swallow }\end{array}$ & H. daurica & 1 & 2 & $\mathrm{R}$ & $\mathrm{LC}$ & $\mathrm{T}$ \\
\hline \multirow[t]{3}{*}{ Lanidae } & Brown Shrike & Lanius cristatus & 2 & 3 & $\mathrm{R}$ & $\mathrm{LC}$ & $\mathrm{T}$ \\
\hline & Long-tailed Shrike & L. schach & 4 & 37 & $\mathrm{UC}$ & $\mathrm{LC}$ & GL, T, US \\
\hline & $\begin{array}{l}\text { Grey-backed } \\
\text { Shrike }\end{array}$ & L. tephronotus & 1 & 1 & $\mathrm{R}$ & $\mathrm{LC}$ & $\mathrm{T}$ \\
\hline \multirow[t]{2}{*}{ Monarchidae } & $\begin{array}{l}\text { Black-naped } \\
\text { Monarch }\end{array}$ & $\begin{array}{l}\text { Hypothymis } \\
\text { azurea }\end{array}$ & 1 & 2 & $\mathrm{R}$ & $\mathrm{LC}$ & $\mathrm{T}$ \\
\hline & $\begin{array}{l}\text { Asian Paradise } \\
\text { Flycatcher }\end{array}$ & $\begin{array}{l}\text { Terpsiphone } \\
\text { paradisi }\end{array}$ & 1 & 1 & $\mathrm{R}$ & $\mathrm{LC}$ & $\mathrm{T}$ \\
\hline \multirow[t]{2}{*}{ Motacillidae } & Olive-backed Pipit & Anthus hodgsoni & 1 & 2 & $\mathrm{R}$ & $\mathrm{LC}$ & GL \\
\hline & Paddyfield Pipit & A. rufulus & 1 & 7 & $\mathrm{R}$ & $\mathrm{LC}$ & GL, RS, US \\
\hline
\end{tabular}




\begin{tabular}{|c|c|c|c|c|c|c|c|}
\hline $\begin{array}{l}\text { Order and } \\
\text { Family name }\end{array}$ & Common Name & Scientific Name & $\mathbf{n}$ & $\begin{array}{c}\text { No. of } \\
\text { Individual }\end{array}$ & $\mathbf{R A}$ & $\begin{array}{l}\text { IUCN } \\
\text { Status }\end{array}$ & Microhabitat \\
\hline & Richard's Pipit & A. richardi & 1 & 3 & $\mathrm{R}$ & LC & GL \\
\hline & Citrine Wagtail & Motacilla citreola & 1 & 18 & $\mathrm{R}$ & $\mathrm{LC}$ & GL, PW \\
\hline & $\begin{array}{l}\text { White-browed } \\
\text { Wagtail }\end{array}$ & $\begin{array}{l}\text { M. } \\
\text { madaraspatensis }\end{array}$ & 1 & 1 & $\mathrm{R}$ & $\mathrm{LC}$ & PW \\
\hline & Yellow Wagtail & M. flava & 1 & 15 & $\mathrm{R}$ & $\mathrm{LC}$ & GL, PW, TW \\
\hline \multirow[t]{11}{*}{ Muscicapidae } & $\begin{array}{l}\text { Common } \\
\text { Stonechat }\end{array}$ & $\begin{array}{l}\text { Saxicola } \\
\text { torquatus }\end{array}$ & 1 & 19 & $\mathrm{R}$ & $\mathrm{LC}$ & GL \\
\hline & Pied Bushchat & S. caprata & 1 & 4 & $\mathrm{R}$ & $\mathrm{LC}$ & GL \\
\hline & $\begin{array}{l}\text { White-tailed } \\
\text { Stonechat }\end{array}$ & S. leucurus & 1 & 2 & $\mathrm{R}$ & $\mathrm{LC}$ & GL \\
\hline & $\begin{array}{l}\text { Oriental Magpie } \\
\text { Robin }\end{array}$ & $\begin{array}{l}\text { Copsychus } \\
\text { saularis }\end{array}$ & 10 & 449 & $\mathrm{VC}$ & $\mathrm{LC}$ & GL, RS, T, US \\
\hline & Taiga Flycatcher & Ficedula albicilla & 1 & 1 & $\mathrm{R}$ & $\mathrm{LC}$ & $\mathrm{T}$ \\
\hline & $\begin{array}{l}\text { Grey-headed } \\
\text { Canary Flycatcher }\end{array}$ & $\begin{array}{l}\text { Culicicapa } \\
\text { ceylonensis }\end{array}$ & 1 & 17 & $\mathrm{R}$ & $\mathrm{LC}$ & $\mathrm{T}$ \\
\hline & $\begin{array}{l}\text { Verditer } \\
\text { Flycatcher }\end{array}$ & $\begin{array}{l}\text { Eumyias } \\
\text { thalassina }\end{array}$ & 1 & 1 & $\mathrm{R}$ & $\mathrm{LC}$ & $\mathrm{T}$ \\
\hline & Black Redstart & $\begin{array}{l}\text { Phoenicurus } \\
\text { ochruros }\end{array}$ & 1 & 1 & $\mathrm{R}$ & $\mathrm{LC}$ & GL \\
\hline & Bluethroat & Luscinia svecica & 1 & 2 & $\mathrm{R}$ & $\mathrm{LC}$ & $\mathrm{T}$ \\
\hline & $\begin{array}{l}\text { Siberian Blue } \\
\text { Robin }\end{array}$ & L. cyane & 1 & 1 & $\mathrm{R}$ & $\mathrm{LC}$ & $\mathrm{T}$ \\
\hline & $\begin{array}{l}\text { Siberian } \\
\text { Rubythroat }\end{array}$ & L. calliope & 1 & 1 & $\mathrm{R}$ & $\mathrm{LC}$ & GL \\
\hline \multirow[t]{2}{*}{ Nectariniidae } & Purple Sunbird & $\begin{array}{l}\text { Nectarinia } \\
\text { asiatica }\end{array}$ & 5 & 31 & $\mathrm{UC}$ & $\mathrm{LC}$ & $\mathrm{T}$ \\
\hline & $\begin{array}{l}\text { Purple-rumped } \\
\text { Sunbird }\end{array}$ & N. zeylonica & 2 & 3 & $\mathrm{R}$ & $\mathrm{LC}$ & $\mathrm{T}$ \\
\hline \multirow[t]{3}{*}{ Oriolidae } & $\begin{array}{l}\text { Black-hooded } \\
\text { Oriole }\end{array}$ & $\begin{array}{l}\text { Oriolus } \\
\text { xanthornus }\end{array}$ & 7 & 77 & $\mathrm{C}$ & $\mathrm{LC}$ & $\mathrm{T}, \mathrm{US}$ \\
\hline & $\begin{array}{l}\text { Black-naped } \\
\text { Oriole }\end{array}$ & O. chinensis & 3 & 8 & $\mathrm{UC}$ & $\mathrm{LC}$ & $\mathrm{T}$ \\
\hline & $\begin{array}{l}\text { Eurasian Golden } \\
\text { Oriole }\end{array}$ & O. oriolus & 1 & 1 & $\mathrm{R}$ & $\mathrm{LC}$ & $\mathrm{T}$ \\
\hline Paridae & Great Tit & Parus major & 2 & 15 & $\mathrm{R}$ & $\mathrm{LC}$ & $\mathrm{T}$ \\
\hline Passeridae & House Sparrow & Passer domesticus & 11 & 1378 & $\mathrm{VC}$ & $\mathrm{LC}$ & GL, RS, T, US \\
\hline Ploceidae & Baya Weaver & $\begin{array}{l}\text { Ploceus } \\
\text { philippinus }\end{array}$ & 3 & 19 & $\mathrm{UC}$ & $\mathrm{LC}$ & $\mathrm{T}$ \\
\hline Rhipiduridae & $\begin{array}{l}\text { White-throated } \\
\text { Fantail }\end{array}$ & $\begin{array}{l}\text { Rhipidura } \\
\text { albicollis }\end{array}$ & 1 & 1 & $\mathrm{R}$ & $\mathrm{LC}$ & $\mathrm{T}$ \\
\hline \multirow[t]{2}{*}{ Pycnonotidae } & $\begin{array}{l}\text { Red-whiskered } \\
\text { Bulbul }\end{array}$ & $\begin{array}{l}\text { Pycnonotus } \\
\text { jocosus }\end{array}$ & 1 & 1 & $\mathrm{R}$ & $\mathrm{LC}$ & $\mathrm{T}$ \\
\hline & Red-vented Bulbul & P. cafer & 11 & 442 & $\mathrm{VC}$ & $\mathrm{LC}$ & GL, T, US \\
\hline \multirow[t]{5}{*}{ Sturnidae } & Bank Myna & $\begin{array}{l}\text { Acridotheres } \\
\text { ginginianus }\end{array}$ & 1 & 19 & $\mathrm{R}$ & $\mathrm{LC}$ & GL, PW, TW \\
\hline & Common Myna & A. tristis & 10 & 1099 & $\mathrm{VC}$ & $\mathrm{LC}$ & GL, RS, T,US \\
\hline & Jungle Myna & A. fuscus & 8 & 294 & $\mathrm{C}$ & $\mathrm{LC}$ & GL, T, US \\
\hline & $\begin{array}{l}\text { Asian Pied } \\
\text { Starling } \\
\end{array}$ & Sturnus contra & 11 & 1079 & $\mathrm{VC}$ & $\mathrm{LC}$ & GL, RS, T,US \\
\hline & $\begin{array}{l}\text { Chestnut-tailed } \\
\text { Starling }\end{array}$ & S. malabaricus & 9 & 262 & $\mathrm{VC}$ & $\mathrm{LC}$ & GL, T, US \\
\hline
\end{tabular}




\begin{tabular}{|c|c|c|c|c|c|c|c|}
\hline $\begin{array}{l}\text { Order and } \\
\text { Family name } \\
\end{array}$ & Common Name & Scientific Name & $\mathbf{n}$ & $\begin{array}{c}\text { No. of } \\
\text { Individual }\end{array}$ & $\mathbf{R A}$ & $\begin{array}{l}\text { IUCN } \\
\text { Status } \\
\end{array}$ & Microhabitat \\
\hline \multirow[t]{5}{*}{ Sylviidae } & Dusky Warbler & $\begin{array}{l}\text { Phylloscopus } \\
\text { fuscatus }\end{array}$ & 1 & 7 & $\mathrm{R}$ & $\mathrm{LC}$ & PW, T \\
\hline & $\begin{array}{l}\text { Clamorous } \\
\text { Reedwarbler }\end{array}$ & $\begin{array}{l}\text { Acrocephalus } \\
\text { stentoreus }\end{array}$ & 1 & 11 & $\mathrm{R}$ & $\mathrm{LC}$ & $\mathrm{T}$ \\
\hline & $\begin{array}{l}\text { Common } \\
\text { Tailorbird }\end{array}$ & $\begin{array}{l}\text { Orthotomus } \\
\text { sutorius }\end{array}$ & 8 & 123 & $\mathrm{C}$ & $\mathrm{LC}$ & $\mathrm{T}$ \\
\hline & Striated Grassbird & $\begin{array}{l}\text { Megalurus } \\
\text { palustris }\end{array}$ & 1 & 7 & $\mathrm{R}$ & $\mathrm{LC}$ & GL, T \\
\hline & $\begin{array}{l}\text { Blyth's Reed } \\
\text { Warbler }\end{array}$ & $\begin{array}{l}\text { Acrocephalus } \\
\text { dumetorum }\end{array}$ & 2 & 7 & $\mathrm{R}$ & $\mathrm{LC}$ & $\mathrm{T}$ \\
\hline \multirow[t]{2}{*}{ Timaliidae } & Striated Babbler & Turdoides earlei & 1 & 2 & $\mathrm{R}$ & $\mathrm{LC}$ & GL \\
\hline & Jungle Babbler & T. striata & 2 & 46 & $\mathrm{R}$ & $\mathrm{LC}$ & GL, T \\
\hline \multirow[t]{2}{*}{ Turdidae } & $\begin{array}{l}\text { Eurasian Scaly } \\
\text { Thrush }\end{array}$ & Zoothera dauma & 2 & 2 & $\mathrm{R}$ & $\mathrm{LC}$ & $\mathrm{T}$ \\
\hline & $\begin{array}{l}\text { Orange-headed } \\
\text { Thrush }\end{array}$ & Z. citrina & 1 & 5 & $\mathrm{R}$ & $\mathrm{LC}$ & $\mathrm{T}$ \\
\hline Zestropodidae & $\begin{array}{l}\text { Oriental White- } \\
\text { eye }\end{array}$ & $\begin{array}{l}\text { Zosterops } \\
\text { palpebrosus }\end{array}$ & 1 & 12 & $\mathrm{R}$ & $\mathrm{LC}$ & $\mathrm{T}$ \\
\hline \multirow[t]{2}{*}{$\begin{array}{l}\text { Gruiformes } \\
\text { Rallidae }\end{array}$} & Common Moorhen & $\begin{array}{l}\text { Gallinula } \\
\text { chloropus }\end{array}$ & 1 & 3 & $\mathrm{R}$ & $\mathrm{LC}$ & PW, TW \\
\hline & $\begin{array}{l}\text { White-breasted } \\
\text { Waterhen }\end{array}$ & $\begin{array}{l}\text { Amaurornis } \\
\text { phoenicurus }\end{array}$ & 2 & 10 & $\mathrm{R}$ & $\mathrm{LC}$ & $\mathrm{PW}, \mathrm{T}, \mathrm{TW}$ \\
\hline \multirow[t]{9}{*}{$\begin{array}{l}\text { Cuculiformes } \\
\text { Cuculidae }\end{array}$} & Asian Koel & $\begin{array}{l}\text { Eudynamys } \\
\text { scolopaceus }\end{array}$ & 7 & 60 & $\mathrm{C}$ & $\mathrm{LC}$ & $\mathrm{T}, \mathrm{US}$ \\
\hline & Indian Cuckoo & $\begin{array}{l}\text { Cuculus } \\
\text { micropterus }\end{array}$ & 1 & 4 & $\mathrm{R}$ & $\mathrm{LC}$ & $\mathrm{T}$ \\
\hline & Common Cuckoo & C. canorus & 1 & 4 & $\mathrm{R}$ & $\mathrm{DD}$ & $\mathrm{T}$ \\
\hline & $\begin{array}{l}\text { Common Hawk } \\
\text { Cuckoo }\end{array}$ & $\begin{array}{l}\text { Hierococcyx } \\
\text { varius }\end{array}$ & 1 & 3 & $\mathrm{R}$ & $\mathrm{LC}$ & $\mathrm{T}$ \\
\hline & Plaintive Cuckoo & $\begin{array}{l}\text { Cacomantis } \\
\text { merulinus }\end{array}$ & 4 & 6 & $\mathrm{UC}$ & $\mathrm{LC}$ & $\mathrm{T}$ \\
\hline & Jacobin Cuckoo & $\begin{array}{l}\text { Clamator } \\
\text { jacobinus }\end{array}$ & 2 & 4 & $\mathrm{R}$ & $\mathrm{LC}$ & $\mathrm{T}$ \\
\hline & $\begin{array}{l}\text { Green-billed } \\
\text { Malkoha }\end{array}$ & $\begin{array}{l}\text { Phaenicophaeus } \\
\text { tristis }\end{array}$ & 1 & 2 & $\mathrm{R}$ & $\mathrm{LC}$ & $\mathrm{T}$ \\
\hline & Greater Coucal & $\begin{array}{l}\text { Centropus } \\
\text { sinensis }\end{array}$ & 2 & 6 & $\mathrm{R}$ & $\mathrm{LC}$ & $\mathrm{T}$ \\
\hline & Lesser Coucal & C. bengalensis & 1 & 2 & $\mathrm{R}$ & $\mathrm{LC}$ & GL \\
\hline \multirow[t]{10}{*}{$\begin{array}{l}\text { Pelecaniformes } \\
\text { Ardeidae }\end{array}$} & $\begin{array}{l}\text { Black-crowned } \\
\text { Night Heron }\end{array}$ & $\begin{array}{l}\text { Nycticorax } \\
\text { nycticorax }\end{array}$ & 2 & 71 & $\mathrm{R}$ & $\mathrm{LC}$ & $\mathrm{T}$ \\
\hline & Black Bittern & $\begin{array}{l}\text { Ixobrychus } \\
\text { flavicollis }\end{array}$ & 1 & 1 & $\mathrm{R}$ & NT & TW \\
\hline & Cinnamon Bittern & I. cinnamomeus & 1 & 6 & $\mathrm{R}$ & $\mathrm{LC}$ & GL \\
\hline & Yellow Bittern & I. sinensis & 1 & 9 & $\mathrm{R}$ & $\mathrm{LC}$ & $\begin{array}{c}\text { GL, PW, TW, } \\
\text { US }\end{array}$ \\
\hline & Cattle Egret & Bubulcus ibis & 1 & 1 & $\mathrm{R}$ & $\mathrm{LC}$ & GL \\
\hline & Intermediate Egret & Ardea intermedia & 1 & 1 & $\mathrm{R}$ & $\mathrm{LC}$ & PW \\
\hline & Little Egret & Egretta garzetta & 3 & 9 & $\mathrm{UC}$ & $\mathrm{LC}$ & GL, PW \\
\hline & Great White Egret & Ardea alba & 1 & 8 & $\mathrm{R}$ & $\mathrm{LC}$ & PW, TW \\
\hline & Purple Heron & A. purpurea & 1 & 1 & $\mathrm{R}$ & $\mathrm{LC}$ & TW \\
\hline & Grey Heron & A. cinerea & 1 & 90 & $\mathrm{R}$ & $\mathrm{LC}$ & PW, TW \\
\hline
\end{tabular}




\begin{tabular}{|c|c|c|c|c|c|c|c|}
\hline $\begin{array}{l}\text { Order and } \\
\text { Family name }\end{array}$ & Common Name & Scientific Name & $\mathbf{n}$ & $\begin{array}{c}\text { No. of } \\
\text { Individual }\end{array}$ & $\mathbf{R A}$ & $\begin{array}{l}\text { IUCN } \\
\text { Status }\end{array}$ & Microhabitat \\
\hline & Little Heron & Butorides striata & 3 & 5 & $\mathrm{UC}$ & $\mathrm{LC}$ & GL, PW, T \\
\hline & Indian Pond Heron & Ardeola grayii & 9 & 91 & $\mathrm{VC}$ & $\mathrm{LC}$ & GL, PW, T, TW \\
\hline \multirow{5}{*}{$\begin{array}{l}\text { Piciformes } \\
\text { Picidae }\end{array}$} & Eurasian Wryneck & Jynx torquilla & 1 & 6 & $\mathrm{R}$ & $\mathrm{LC}$ & GL, T \\
\hline & $\begin{array}{l}\text { Black-rumped } \\
\text { Flameback }\end{array}$ & $\begin{array}{l}\text { Dinopium } \\
\text { benghalense }\end{array}$ & 9 & 80 & $\mathrm{VC}$ & $\mathrm{LC}$ & GL, RS, T \\
\hline & Greater Flameback & $\begin{array}{l}\text { Chrysocolaptes } \\
\text { guttacristatus }\end{array}$ & 1 & 11 & $\mathrm{R}$ & $\mathrm{LC}$ & $\mathrm{T}$ \\
\hline & $\begin{array}{l}\text { Fulvous-breasted } \\
\text { Woodpecker }\end{array}$ & $\begin{array}{l}\text { Dendrocopos } \\
\text { macei }\end{array}$ & 7 & 45 & $\mathrm{C}$ & $\mathrm{LC}$ & $\mathrm{T}, \mathrm{US}$ \\
\hline & $\begin{array}{l}\text { Rufous } \\
\text { Woodpecker }\end{array}$ & $\begin{array}{l}\text { Micropternus } \\
\text { brachyurus }\end{array}$ & 2 & 12 & $\mathrm{R}$ & LC & $\mathrm{T}$ \\
\hline \multirow[t]{3}{*}{ Megalaimidae } & $\begin{array}{l}\text { Blue-throated } \\
\text { Barbet }\end{array}$ & $\begin{array}{l}\text { Psilopogon } \\
\text { asiaticus }\end{array}$ & 1 & 2 & $\mathrm{R}$ & $\mathrm{LC}$ & $\mathrm{T}$ \\
\hline & Lineated Barbet & P. lineatus & 2 & 19 & $\mathrm{R}$ & $\mathrm{LC}$ & $\mathrm{T}$ \\
\hline & $\begin{array}{l}\text { Coppersmith } \\
\text { Barbet }\end{array}$ & P. haemacephalus & 7 & 87 & $\mathrm{C}$ & $\mathrm{LC}$ & $\mathrm{T}$ \\
\hline $\begin{array}{l}\text { Podicipediformes } \\
\text { Podicipedidae }\end{array}$ & Little Grebe & $\begin{array}{l}\text { Tachybaptus } \\
\text { ruficollis }\end{array}$ & 1 & 4 & $\mathrm{R}$ & $\mathrm{LC}$ & PW \\
\hline \multirow{5}{*}{$\begin{array}{l}\text { Strigiformes } \\
\text { Strigidae }\end{array}$} & Brown Boobook & Ninox scutulata & 1 & 1 & $\mathrm{R}$ & $\mathrm{LC}$ & $\mathrm{T}$ \\
\hline & Brown Fish Owl & Ketupa zeylonensis & 1 & 1 & $\mathrm{R}$ & $\mathrm{LC}$ & $\mathrm{T}$ \\
\hline & $\begin{array}{l}\text { Collared Scops } \\
\text { Owl }\end{array}$ & Otus lettia & 1 & 1 & $\mathrm{R}$ & $\mathrm{LC}$ & $\mathrm{T}$ \\
\hline & Short Eared Owl & Asio flammeus & 1 & 1 & $\mathrm{R}$ & LC & $\mathrm{T}$ \\
\hline & Spotted Owlet & Athene brama & 3 & 11 & $\mathrm{UC}$ & $\mathrm{LC}$ & $\mathrm{T}$ \\
\hline Tytonidae & $\begin{array}{l}\text { Common Barn } \\
\text { Owl }\end{array}$ & Tyto alba & 1 & 1 & $\mathrm{R}$ & $\mathrm{LC}$ & US \\
\hline \multirow[t]{2}{*}{$\begin{array}{l}\text { Suliformes } \\
\text { Phalacrocoracidae }\end{array}$} & Great Cormorant & $\begin{array}{l}\text { Phalacrocorax } \\
\text { carbo }\end{array}$ & 1 & 8 & $\mathrm{R}$ & $\mathrm{LC}$ & PW \\
\hline & Little Cormorant & Microcarbo niger & 10 & 154 & $\mathrm{VC}$ & $\mathrm{LC}$ & $\begin{array}{c}\text { GL, PW, T, TW, } \\
\text { US }\end{array}$ \\
\hline $\begin{array}{l}\text { Ciconiiformes } \\
\text { Ciconiidae } \\
\end{array}$ & Asian Openbill & $\begin{array}{l}\text { Anastomus } \\
\text { oscitans }\end{array}$ & 1 & 4 & $\mathrm{R}$ & $\mathrm{LC}$ & $\mathrm{T}$ \\
\hline \multirow[t]{8}{*}{$\begin{array}{l}\text { Columbiformes } \\
\text { Columbidae }\end{array}$} & $\begin{array}{l}\text { Orange-breasted } \\
\text { Green Pigeo }\end{array}$ & Treron bicinctus & 1 & 1 & $\mathrm{R}$ & $\mathrm{LC}$ & $\mathrm{T}$ \\
\hline & $\begin{array}{l}\text { Yellow footed } \\
\text { Green Pigeon }\end{array}$ & T. phoenicopterus & 1 & 3 & $\mathrm{R}$ & $\mathrm{LC}$ & $\mathrm{T}$ \\
\hline & $\begin{array}{l}\text { Eurasian Collared } \\
\text { Dove }\end{array}$ & $\begin{array}{l}\text { Streptopelia } \\
\text { decaocto }\end{array}$ & 3 & 11 & $\mathrm{UC}$ & $\mathrm{LC}$ & $\mathrm{T}, \mathrm{US}$ \\
\hline & Red Turtle Dove & S. tranquebarica & 1 & 7 & $\mathrm{R}$ & $\mathrm{LC}$ & $\mathrm{T}, \mathrm{US}$ \\
\hline & $\begin{array}{l}\text { Green Imperial } \\
\text { Pigeon }\end{array}$ & Ducula aenea & 1 & 1 & $\mathrm{R}$ & $\mathrm{LC}$ & $\mathrm{T}$ \\
\hline & $\begin{array}{l}\text { Grey-capped } \\
\text { Emerald Dove }\end{array}$ & $\begin{array}{l}\text { Chalcophaps } \\
\text { indica }\end{array}$ & 1 & 1 & $\mathrm{R}$ & $\mathrm{LC}$ & GL \\
\hline & Rock Pigeon & Columba livia & 8 & 124 & $\mathrm{C}$ & $\mathrm{LC}$ & GL,RS, T, US \\
\hline & Spotted Dove & $\begin{array}{l}\text { Spilopelia } \\
\text { chinensis }\end{array}$ & 10 & 222 & $\mathrm{VC}$ & $\mathrm{LC}$ & GL, RS, T, US \\
\hline \multicolumn{8}{|c|}{ Mammalia } \\
\hline $\begin{array}{l}\text { Carnivora } \\
\text { Canidae }\end{array}$ & Goden Jackal & Canis aureus & 1 & 1 & $\mathrm{R}$ & $\mathrm{LC}$ & GL \\
\hline Herpestidae & $\begin{array}{l}\text { Common } \\
\text { Mongoose }\end{array}$ & $\begin{array}{l}\text { Herpestes } \\
\text { edwardsii }\end{array}$ & 5 & 15 & $\mathrm{R}$ & $\mathrm{LC}$ & GL, T, US \\
\hline
\end{tabular}




\begin{tabular}{|c|c|c|c|c|c|c|c|}
\hline $\begin{array}{l}\text { Order and } \\
\text { Family name }\end{array}$ & Common Name & Scientific Name & $\mathbf{n}$ & $\begin{array}{c}\text { No. of } \\
\text { Individual }\end{array}$ & RA & $\begin{array}{l}\text { IUCN } \\
\text { Status }\end{array}$ & Microhabitat \\
\hline Felidae & Jungle Cat & Felis chaus & 1 & 1 & $\mathrm{R}$ & NT & GL \\
\hline \multirow[t]{3}{*}{ Viverridae } & $\begin{array}{l}\text { Common Palm } \\
\text { Civet }\end{array}$ & $\begin{array}{l}\text { Paradoxurus } \\
\text { hermaphroditus }\end{array}$ & 1 & 1 & $\mathrm{R}$ & $\mathrm{LC}$ & GL \\
\hline & Large Indian Civet & Viverra zibetha & 2 & 2 & $\mathrm{R}$ & NT & GL \\
\hline & Small Indian Civet & Viverricula indica & 2 & 2 & $\mathrm{R}$ & NT & GL \\
\hline $\begin{array}{l}\text { Cetartiodactyla } \\
\text { Platanistidae }\end{array}$ & $\begin{array}{l}\text { Ganges River } \\
\text { Dolphin }\end{array}$ & $\begin{array}{l}\text { Platanista } \\
\text { gangetica }\end{array}$ & 1 & 3 & $\mathrm{R}$ & VU & PW \\
\hline \multirow[t]{2}{*}{$\begin{array}{l}\text { Chiroptera } \\
\text { Pteropodidae }\end{array}$} & $\begin{array}{l}\text { Greater Short- } \\
\text { nosed } \\
\text { fruit Bat }\end{array}$ & $\begin{array}{l}\text { Cynopterus } \\
\text { sphinx }\end{array}$ & 3 & 15 & $\mathrm{R}$ & LC & $\mathrm{T}$ \\
\hline & Indian Flying Fox & $\begin{array}{l}\text { Pteropus } \\
\text { giganteus }\end{array}$ & 6 & 417 & $\mathrm{VC}$ & $\mathrm{LC}$ & GL, US \\
\hline Vespertilionidae & Indian Pipistrelle & $\begin{array}{l}\text { Pipistrellus } \\
\text { coromandra }\end{array}$ & 1 & 1 & $\mathrm{R}$ & LC & $\mathrm{T}$ \\
\hline \multirow[t]{2}{*}{$\begin{array}{l}\text { Rodentia } \\
\text { Sciuridae }\end{array}$} & Irrawaddy Squirrel & $\begin{array}{l}\text { Callosciurus } \\
\text { pygerythrus }\end{array}$ & 3 & 40 & $\mathrm{UC}$ & $\mathrm{LC}$ & $\mathrm{T}$ \\
\hline & $\begin{array}{l}\text { Five-striped Palm } \\
\text { Squirrel }\end{array}$ & $\begin{array}{l}\text { Funambulus } \\
\text { pennantii }\end{array}$ & 4 & 108 & $\mathrm{VC}$ & $\mathrm{LC}$ & GL, T, US \\
\hline \multirow[t]{2}{*}{ Muridae } & $\begin{array}{l}\text { Greater Bandicoot } \\
\text { Rat }\end{array}$ & $\begin{array}{l}\text { Bandicota } \\
\text { bengalensis }\end{array}$ & 1 & 1 & $\mathrm{R}$ & $\mathrm{LC}$ & GL \\
\hline & $\begin{array}{l}\text { Lesser Bandicoot } \\
\text { Rat }\end{array}$ & B. indica & 2 & 3 & $\mathrm{R}$ & $\mathrm{LC}$ & GL, US \\
\hline $\begin{array}{l}\text { Eulipotyphla } \\
\text { Soricidae }\end{array}$ & House Shrew & Suncus murinus & 3 & 9 & $\mathrm{R}$ & LC & GL \\
\hline $\begin{array}{l}\text { Primates } \\
\text { Cercopithecidae }\end{array}$ & Rhesus Macaque & Macaca mulatta & 2 & 253 & $\mathrm{VC}$ & $\mathrm{VU}$ & $\mathrm{T}$ \\
\hline
\end{tabular}

Table 3. List of wildlife (Amphibia to Mammalia) observed in the urban Dhaka megacity. (n: Frequency of Occurrence, RA: Relative Abundance, VC: Very Common; C: Common, UC: Uncommon, R: Rare, LC: Least Concern, NT: Near Threatened, VU: Vulnerable, DD: Data Deficient, GL: Grassland, PW: Permanent Waterbody, TW: Temporary Waterbody, RS: Roadside, US: Urban Settlement, T: Tree).

than in the earlier study. But, we have two new locality distribution records for amphibian species (Kalasgram Skipper Frog and Yellow Striped Frog) not previously reported by Reza \& Perry (2015). We also recorded four frog species in the city under the genus Fejervarya, among six recorded across Bangladesh.

Reptiles were the least observed wildlife, found only in eight sites among the eleven surveyed. Among the reptiles, the highest species diversity was found for the family Gekkonidae ( $21 \%, 4$ species). As for species compositions, almost half of the species were snakes $(47.4 \%, 9$ species) and the rest were lizards and turtles. More importantly, this study found two species of venomous snakes (Monocled Cobra and Binocled Cobra) in the grassland. No other published reports about herpetofauna in Dhaka city are available. This study found 31 species of herpetofauna. However, Reza \& Perry (2015) found 31 species of amphibians and reptiles in the Jahangirnagar University Campus (Savar), a neighboring location of Dhaka city. They reported Ornate Flying Snake, Wall's Bronzeback, Bowring's House Gecko, Diard's Blind Snake, Jerdon's Blind Snake and Streaked Kukri Snake, which were not observed at our study sites. On the other hand, we recorded new species distribution for seven reptiles (White-spotted Supple Skink, Monocled Cobra, Roofed Turtle, Common Wolf Snake, Common Blind Snake, Common Bronzeback Tree Snake and Checkered Keelback).

Species diversity of birds was the highest for the family Ardeidae $(8 \%, 13$ species). The order Passeriformes was the most diverse order representing 72 species of birds. This study recorded some rare species that usually occur in forests, such as the Crested Serpent Eagle, Alexandrine Parakeet, Plum-headed Parakeet, Red-breasted Parakeet, Lesser Racket-tailed Drongo, Ashy Woodswallow, 
Large Woodshrike, Red-whiskered Bulbul, Greenbilled Malkoha, Lesser Coucal, Collared Scops owl, Orange-breasted Green Pigeon, Green Imperial Pigeon and Grey-capped Emerald Dove. This is possible because these rare birds were recorded from peripheral habitats of Dhaka megacity and the Mirpur National Botanical Garden. NBG is a protected area of the Bangladesh Forest Department that is regulated by the Bangladesh Wildlife (Conservation and Security) Act, 2012 that probably facilitates the presence of many species of wildlife, particularly forest birds. It is understood that birds can move easily between habitats, so they prefer to aggregate in such habitats where food and nesting trees are available. Our results suggest that the regional abundance of birds depends heavily on areas in which the traditional landscape and vegetation remain relatively intact. In addition, we also assumed that these forest birds may migrate from neighboring protected areas like Bhawal National Park and Modhupur National Park, about $20 \mathrm{~km}$ and $50 \mathrm{~km}$ away from Dhaka city, respectively, while flying over the Dhaka city.

Birds were seen easily in all study sites. This may be because some species prefer to colonize homestead urban habitats, for example, the Common Myna, House Crow, and Oriental Magpie Robin. In some study sites such as the Dhaka University campus, Ramna park, Uttara and Mirpur were represented by many species of birds. Uttara represented diverse habitats that were less disturbed and utilized by some important species of bird as foraging sites. We observed 34 species of birds in Shahbag near the Dhaka University campus. In the same study site, Akash et al. (2013) found 50 species and Chowdhury et al. (2014) found 78 species. We also observed 34 species of birds in Ramna park where Rajia et al. (2015) previously found 50 species. Avian richness in Shahbagh and Ramna has decreased from previous years, probably due to the impact of anthropogenic factors (e.g. pollution, construction work, human pressure). In Uttara study site, we found 125 species of birds, but Sarker et al. (2009) reported only 27 species from 2 out of 17 sectors of Uttara. In Uttara, particularly in Diabari (a fragmented place of Uttara), there are many planted trees, which enrich the homestead forests, and some fallow lands, grasslands and aquatic bodies including ponds that provide suitable habitats for wildlife. The diversity of avifauna has been decreasing gradually in most of the locations of Dhaka

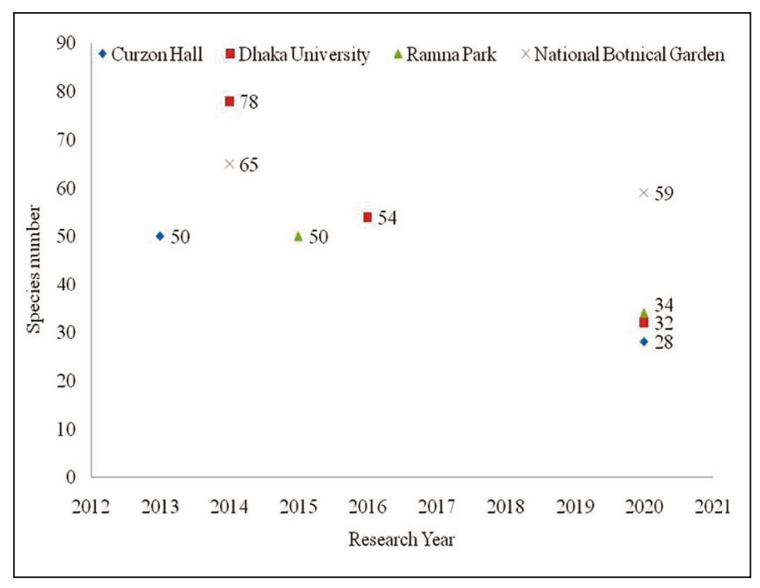

Figure 5. Trends of avifauna in the urban Dhaka megacity, present Study - Curzon Hall, Dhaka University, Ramna Park and National Botanical Garden; Akash et al. (2013); Chowdhury et al. (2014); Islam et al. (2014); Rajia et al. (2015); Banu et al. (2016).

city, particularly on the Dhaka University Campus (Fig. 5). However, this study recorded 24 colonies for bird species not reported before.

Among mammals, one-third of the total mammalian species were under the order Carnivora (37.5\%, 6 species) followed by the order Rodentia (25\%, 4 species). Mammalian fauna, especially the Flying Fox ( $47.8 \%, 417$ indiv.), was frequently found in Dhaka city, particularly in the Ramna park area; almost half of the observed mammalian individuals. Ramna park is the only site where large fruiting, resting and roosting trees are available, which facilitate Flying Fox to roam inside the park. Furthermore, Ramna park is managed and protected by the local administration, which might be the reason for the highest number of bats being sighted there. Urban habitats of Dhaka have a few patchy areas that can support urban mammals. We found some small mammals like the Common Mongoose, Five Striped Palm Squirrel in Old Dhaka. These small mammals are well adapted in urban habitats and use bushes and trees to hide. The habitat of Old Dhaka is suitable for these small mammals. We also found that the Gangetic River Dolphin occurred in Buriganga river near Bosila and that the Jungle Cat and Golden Jackal were only found in the riverside of Buriganga. Buriganga is connected with the Meghna river via Dhaleswari which is used by the Gangetic River Dophin (IUCN Bangladesh, 2015). The riverside bushy areas are the ideal habitat for Jungle Cat and Golden Jackal. The other study 
sites which we studied did not include this type of combined habitat hence they are seen here. We found a non-human primate, Rhesus Macaque in Gandaria within the Old Dhaka site.

\section{Diversity indices}

Diversity indices are important tools for priority settings to conserve species. The Shannon-Wiener index of diversity showed the species diversity of different study sites. Species diversity was rich in the sites where enriched floral diversity near the homestead and many waterbodies were present. Species richness is used in the similarity index to avoid species abundance to compare common species found in two sites. The highest number of shared species was found for Mirpur-Uttara $(\mathrm{n}=48)$ and the lowest number was found in Demra-Gulshan areas. The habitat types in Mirpur and Uttara are similar to some extent (Table 1). For this reason, similarity was the highest for these sites indicating similar characteristics or closely related types of habitats supporting those urban wildlife species. This may be true because urban green spaces have been found to greatly influence species colonization and persistence rates (Gallo et al., 2017). In addition, 'Island Theory' may be applicable for explaining our species richness results. For example, Mirpur and Uttara sites, like islands were less complex and more numerous than larger ecosystems. However, we have less opportunity to explain our data on species richness under this theory since the current study was conducted on a terrestrial ecosystem. Interestingly, Uttara and Mirpur, both larger-sized sites in our study area, have characteristics of larger islands, harboring more species than smaller sites like Demra and Gulshan, and Mohammadpur and Demra had fewer species and were more isolated from other sites. Our data about these species richness has been supported by the prediction of MacArthur \& Wilson (2001) that insular species richness depends on island size and isolation from source regions.

\section{Habitat and substrate utilization}

The Common Tree Frog and Yellow Stripped Tree Frog were found in some trees. This means that urban trees may support some arboreal amphibians. The Common Tree Frog and Yellow Stripped Tree Frog were observed at man height layer and all other species were seen using the ground layer of gardens and patchy habitats such as grassland, permanent waterbodies like ponds and rivers, temporary waterbodies like canals and drains, roadside vegetation and urban settlements. Reptilian species were found in arboreal habitats except for the Checkered Keelback and Striped Keelback, which were found in permanent as well as temporary waterbodies. The Indian Flap-Shell Turtle and Indian Roofed Turtle were found in temporary waterbody. Since reptilians are cryptic they change their habitats more frequently than others. In addition, reptilian diversity is known to be the lowest in urbanized areas for several reasons (Gibbon et al., 2000; Mitchell et al., 2008; Todd et al., 2010). This supports our results that reptiles may not prefer urban and disturbed habitats.

Birds used homestead trees, grasslands, even waterbodies for feeding, breeding and also to protect their territory. They were found in all types of layers from the ground level to the upper canopy. The Asian Palm Swift, Black Kite and House Swift were observed flying in all the study sites.

The highest species richness for mammals was found in terrestrial habitats, which includes grasslands, urban settlement and roadside vegetation. Squirrels, shrews, bats and macaque used different canopy layers because of their arboreal and aerial adaptations. Mammals are more susceptible than birds to the physical barriers that characterize the urban matrix, such as roads, buildings, artificial waterways, and increased human activity (Crooks, 2002; McKinney, 2006; Ordeñana et al., 2010).

\section{Identification of anthropogenic threats}

Unmanaged sewage systems and waste dumping pose serious threats to most wildlife habitats. We found that industrial and household polluted water were being directly discharged into city lakes, ponds, canals and nearby rivers without any treatment. Wastes materials such as plastics, polythenes, styrofoam food boxes, chips packets and other garbage were found disposed of openly in aquatic and terrestrial habitats. We identified construction works in progress that was potentially destroying wildlife habitat and hampering the migration route of different species. Random vehicle movements, use of parks and gardens as 
a short passageway were also identified during the study period. Previous studies also identified pollution, mismanagement of waste materials, drainage problems as threats for Dhaka city (Hasan \& Mulamoottil, 1994; Ahmad, 2009; Alom \& Khan, 2014). These factors are known to adversely impact wildlife populations in urban communities (Emlen, 1974). Especially amphibians and other aquatic species are most likely to be affected as new infrastructure fills existing aquatic habitats and cut-off linkages between aquatic habitats. Thus, normal breeding activities of wildlife may be hampered due to such anthropogenic disturbances.

In conclusion, urban and suburban habitats are becoming increasingly important to biodiversity conservation efforts. This is obvious, as this study found rich vertebrate wildlife diversity, including some rare forest birds in the urban habitat. Natural habitats for wildlife in the urban Dhaka megacity have gradually been damaged (Banu et al., 2016, Akash et al., 2018), consequently, urban areas possess a great potential for being considered as future conservation areas (Magle et al., 2012). This study suggests that further research is important to understand how and which anthropogenic factors affect wildlife in the Dhaka megacity. Similarly, city architects and city administration need to understand the ecological effects of urbanization and make plans in line with protecting urban biodiversity.

In closing, we would like to encourage urban planners, decision-makers, ecologists and wildlife biologists to work together to formulate conservation plans to protect the urban wildlife and to make an eco-friendly urban megacity that is good for both humans and wildlife.

\section{ACKNOWLEDGEMENTS}

We are grateful to many anonymous volunteers of the Department of Zoology, University of Dhaka and Jagannath University, Dhaka for their great contribution in field data collection. We are also grateful to the Chairman of the Department of Zoology, University of Dhaka for allowing the space during the research work. We are grateful to Dr. Michael A. Huffman, Associate Professor of Primate Research Institute, Kyoto University, Japan for critically scrutinizing the manuscript and improving the writing.
GRANTS-IN-AID. Some parts of the study were funded by UGC. The rest was funded by the Ministry of Science and Technology, Government of the People's Republic of Bangladesh.

\section{REFERENCES}

Ahmad S., 2009. The Deterioration of Water Bodies in Dhaka City \& Their Impact upon the Environmental Situation of Dhaka City. Bangladesh Journal of Geology, 26: 28.

Akash M., Akter J., Tamanna T. \& Kabir M.R., 2018. The Urbanization and Environmental Challenges in Dhaka City.7th International RAIS Conference on Social Sciences. Available at SSRN: https://ssrn.com/abstract $=3152116$ or

Akash M., Hossain M.A., Chowdhury G.W., Mahmud H. \& Islam M.A., 2013. Status of avifauna in Curzon Hall premises University of Dhaka, Bangladesh. Ecoprint: An International Journal of Ecology, 20: 1-8. https://doi.org/10.3126/eco.v20i0.11324

Alom M.M. \& Khan M.Z.H., 2014. Environmental and social impact due to urban drainage problems in Dhaka City, Bangladesh. International Journal of Engineering and Advanced Technology, 3: 128-132.

Aronson M.F., Lepczyk C.A., Evans K.L., Goddard M.A., Lerman S.B., MacIvor J.S. \& Vargo T., 2017. Biodiversity in the city: key challenges for urban green space management. Frontiers in Ecology and the Environment, 15: 189-196. https://doi.org/10.1002/fee.1480

Banu M.F.A., Akash M., Chowdhury G. W. \& Islam M. A. 2016. Status and seasonal occurrence of birds in Dhaka University Campus. Dhaka University Journal of Biological Science, 25: 27-37. https://doi.org/10.3329/dujbs.v25i1.28492

Bolitzer B. \& Netusil N.R., 2000. The impact of open spaces on property values in Portland, Oregon. Journal of environmental management, 59: 185-193. https://doi.org/10.1006/jema.2000.0351

Chao A., Chazdon R.L., Colwell R.K. \& Shen, T.J., 2005. A new statistical approach for assessing similarity of species composition with incidence and abundance data. Ecology Letters, 8: 148-159. https://doi.org/10.1111/j.1461-0248.2004.00707.x

Chowdhury S., Aich U. \& Shahadat O., 2014. Checklist of avian fauna of Dhaka University Campus, Bangladesh. International Journal of Fauna and Biological Studies, 1: 56-60.

Crooks K.R., 2002. Relative Sensitivities of Mammalian Carnivores to Habitat Fragmentation. Conservation biology, 16: 488-502. https://doi.org/10.1046/j.1523-1739.2002.00386.x 
Crooks K.R., Suarez A.V. \& Bolger D.T., 2004. Avian assemblages along a gradient of urbanization in a highly fragmented landscape. Biological Conservation, 115: 451-462. https://doi.org/10.1016/S0006-3207(03)00162-9

Emlen J.T., 1974. An urban bird community in Tucson, Arizona:derivation, structure, regulation. Condor, 76: 184-197. DOI: 10.2307/1366729

Fitz Gibbon S.I., Putland D.A. \& Goldizen A.W., 2007. The importance of functional connectivity in the conservation of a ground-dwelling mammal in an urban Australian landscape. Landscape Ecology, 22: $1513-1525$ https://doi.org/10.1007/s10980-007-9139-x

Frost D.R., 2020. Amphibian Species of the World: an Online Reference. Version 6.1 (Access on September 2018). Electronic Database accessible at https://amphibiansoftheworld.amnh.org/index.php. American Museum of Natural History, New York, USA.

Gallo T., Fidino M., Lehrer E.W. \& Magle S.B., 2017. Mammal diversity and metacommunity dynamics in urban green spaces: implications for urban wildlife conservation. Ecological Applications, 27: 2330-2341. https://doi.org/10.1002/eap.1611

Gent T. \& Gibson S., 2003. Herpetofauna Workers' Manual (revised reprint), Joint Nature Conservation Committee, Peterborough, Pelagic Publications, 3pp.

Gibbon J.W., Scott D.E., Ryan T.J., Buhlmann K.A., Tuberville T.D., Metts B.S., Greene J.L., Mills T., Leiden Y., Poppy S. \& Winne C.T., 2000. The global decline of reptiles, Deja Vu amphibians. Bio Science, 50: 653-666.

Gibbs J.P., Buff M.F. \& Cosentino B.J., 2019. The biological system-Urban wildlife, adaptation, and evolution: Urbanization as a driver of contemporary evolution in Gray Squirrels (Sciurus carolinensis). In: Hall M. \& Balogh S., 2019. Understanding urban ecology. Springer, Cham, 269-286. https://doi.org/10.1007/978-3-030-11259-2_12

Grimm N.B., Faeth S.H., Golubiewski N.E., Redman C.L., Wu J., Bai X. \& Briggs J.M., 2008. Global change and the ecology of cities. Science, 319: 756-760. https://doi.org/10.1126/science.1150195

Halder R.R., 2010. A photographic guide to the bird of Bangladesh. Baikal Teal Publication, Dhaka, Bangladesh, $257 \mathrm{pp}$.

Hasan M.K., Khan M.H. \& Feeroz M.M., 2014. Amphibians and Reptiles of Bangladesh-A Field Guide. Arannayk Fundation, Dhaka, 191 pp.

Hasan S. \& Mulamoottil G., 1994. Environmental problems of Dhaka City: a study of mismanagement. Cities, 11: 195-200.

Hossain M.M. \& Baki M.A., 2015. Present status of preliminary survey on avifauna diversity and distribution in the most polluted river Buriganga, Dhaka, Bangladesh. International Journal of Pure and Applied Zoology, 3: 59-69.

Howlader M.S.A., Nair A. \& Merilä J., 2016. A New Species of Frog (Anura: Dicroglossidae) Discovered from the Mega City of Dhaka. PLoS ONE, 11: e0149597.

https://dx.doi.org/10.1371\%2Fjournal.pone.0149597

Islam M.S. \& Ahmed R., 2011. Land use change prediction in Dhaka city using GIS aided markov chain modelling. Journal of Life and Earth Science, 6: 81-89. http://banglajol.info.index.php/JLES.

Islam M.S., Shahadat O., Kabir M.M., Rashid M.A., Razi H.A., Kamaruzzaman M., Alam S.M.I., Mustafa T. \& Islam M.S., 2014. Avifauna of the national botanical garden of Bangladesh. Journal of Taxonomy and Biodiversity Research, 6: 17-20.

IUCN Bangladesh., 2015. Red List of Bangladesh Volume 2-4: Mammals, Birds, Amphibians and Reptiles. IUCN, International Union for Conservation of Nature, Bangladesh Country Office, Dhaka, Bangladesh.

Jaman M.F., Majumder M.S., Hossain M.S., Rahman M.M. \& Uddin M., 2014. Diversity of Wildlife at Ruhitpur Union, Keraniganj, Dhaka. Journal of Asiatic Society Bangladesh, Science, 40: 295-308. https://doi.org/10.3329/jasbs.v40i2.46027

Jessop T.S., Smissen P., Scheelings F. \& Dempster T., 2012. Demographic and phenotypic effects of human mediated trophic subsidy on a large Australian lizard (Varanus varius): meal ticket or last supper? PLoS one, 7: e34069.

https://dx.doi.org/10.1371\%2Fjournal.pone.0034069

Kato E., Yano Y. \& Ohe Y., 2019. Investigating Gaps in Perception of Wildlife between Urban and Rural Inhabitants: Empirical Evidence from Japan. Sustainability, 11: 4516. https://doi.org/10.3390/su11174516

Khan M.A.R., 2004. Checklist of the herpetofauna of Bangladesh. Cobra, 57: 1-31.

Khan M.A.R., 2015. Wildlife of Bangladesh-checklist and guide. Chayabithi, Purana Paltan, Dhaka 1000, $568 \mathrm{pp}$.

Khan M.M.H., 2018. Photographic Guide to the Wildlife of Bangladesh. Arannayk Foundation, Dhaka, Bangladesh, $488 \mathrm{pp}$.

MacArthur R.H. \& Wilson E.O., 2001. The theory of island biogeography. Princeton, N.J: Princeton University Press, 203 pp.

Magle S.B., Hunt V.M., Vernon M. \& Crooks K.C., 2012. Urban wildlife research: Past, present, and future. Biological Conservation, 155: 23-32. https://doi.org/10.1016/j.biocon.2012.06.018

McCance E.C., Decker D.J., Colturi A.M., Baydack R.K., Siemer W.F., Curtis P. \& Eason T.H., 2017. Impor- 
tance of Urban Wildlife Management in the United States and Canada. Mammal Study, 42: 1-16. https://doi.org/10.3106/041.042.0108

McKinney M.L., 2006. Urbanization as a Major Cause of Biotic Homogenization.Biological Conservation, 127: 247-60. https://doi.org/10.1016/j.biocon.2005.09.005

Mendes W., Balmer K., Kaethler T. \& Rhoads A., 2008. Using land inventories to plan for urban agriculture: experiences from Portland and Vancouver. Journal of the American Planning Association, 74: 435-449. https://doi.org/10.1080/01944360802354923

Mitchell J., Jung R. \& Bartholomew B., 2008. Urban herpetology. Salt Lake City (UT): Society for the Study of Amphibians and Reptiles, 586 pp.

Ordeñana M.A., Crooks K.R., Boydston E.E., Fisher R.N., Lyren L.M., Siudyla S. \& Miles A.K., 2010. Effects of urbanization on carnivore species distribution and richness. Journal of Mammalogy, 91: 13221331. https://doi.org/10.1644/09-MAMM-A-312.1

Pain D.J., Cunningham A.A., Donald P.F., Duckworth J.W., Houston D.C., Katzner T., Parry-Jones J., Poole C., Prakash V., Round P. \& Timmins R., 2003. Causes and effects of temporospatial declines of Gyps vultures in Asia. Conservation Biology, 17: 661-671. https://doi.org/10.1046/j.1523-1739.2003.01740.x

Rajia S., Alam M.M., Chowdhury G.W., Akash M. \& Islam M.A., 2015. Status and diversity of birds of Ramna Park, Dhaka, Bangladesh. Bangladesh Journal of Zoology, 43: 291-301. https://doi.org/10.3329/bjz.v43i2.27399

Rebolo-Ifra N.N., Tella J.L. \& Carrete M., 2017. Urban Conservation Hotspots: Predation Release Allows the Grassland-specialist Burrowing Owl to Perform Better in the City. Scientific Reports, 7: 1-9. https://doi.org/10.1038/s41598-017-03853-z

Reza A.H.M.A. \& Perry G., 2015. Herpetofaunal species richness in the tropical forests of Bangladesh. Asian Journal of Conservation Biology, 4: 100-108.

Rocha E.A. \& Fellowes M.D.E., 2018. Does urbanization explain differences in interactions between an insect herbivore and its natural enemies and mutualists? Urban Ecosystems, 21: 405-417. https://doi.org/10.1007/s11252-017-0727-5
Saenz D., Hall T.L. \& Kwiatkowski M.A., 2015. Effects of urbanization on the occurrence of Batrachochytrium dendrobatidis: do urban environments provide refuge from the amphibian chytrid fungus? Urban Ecosystems, 18: 333-340. https://doi.org/10.1007/s11252-014-0398-4

Sarker M.A.R., 2013. First confirmed record of Lygosoma albopunctata (Reptilia: Squamata: Scincidae) from Bangladesh. Russian Journal of Herpetology, 21: $1-4$. https://doi.org/10.30906/1026-2296-2014-21-1-1-4 Sarker N.J., Sultana D., Jaman M.F. \& Rahman M.K., 2009. Diversity and population of avifauna of two urban sites of Dhaka, Bangladesh. Ecoprint: An International Journal of Ecology, 16: 1-7. https://doi.org/10.3126/eco.v16i0.3464

Sekercioglu C.H., 2002. Impacts of bird watching on human and avian communities. Environmental conservation, 29(3): 282-289. https://doi.org/10.1017/S0376892902000206

Sekercioglu C.H., Daily G.C. \& Ehrlich P.R., 2004. Ecosystem consequences of bird declines. Proceedings of the National Academy of Sciences, 101: 1804218047. https://doi.org/10.1073/pnas.0408049101

Shannon C.E. \& Weaver W., 1949. The Mathematical Theory of Communication. University of Illinois press, Urbana, $117 \mathrm{pp}$.

Todd B.D., Willson J.D. \& Gibbons J.W., 2010. The global status of reptiles and causes of their decline. In: Sparling D.W., Linder G., Bishop C.A. \& Krest S., 2010. Ecotoxicology of amphibians and reptiles. New York, NY: SETAC, pp. 47-67.

Uetz P., Freed P. \& Hošek J., 2019. The Reptile Database, http://www.reptile-database.org, accessed on 22 Sep tember 2018.

vanHeezik Y. \& Seddon P.J., 2012. Accounting for detectability when estimating avian abundance in an urban area. New Zealand Journal of Ecology, 36: 1. http://www.newzealandecology.org/nzje/

Wheater C.P., Bell J.R. \& Cook P.A., 2011. Practical Field Ecology: A Project Guide. John Wiley \& Sons, Ltd, The Atrium, Southern Gate, Chichester, West Sussex, PO19 8SQ, UK, 464 pp. 
. 
